\title{
A Bayesian Approach to Reduced Order Modeling in Catalytic Steam Reforming
}

Evan D. Ford

Follow this and additional works at: https://researchrepository.wvu.edu/etd

\section{Recommended Citation}

Ford, Evan D., "A Bayesian Approach to Reduced Order Modeling in Catalytic Steam Reforming" (2015). Graduate Theses, Dissertations, and Problem Reports. 5608.

https://researchrepository.wvu.edu/etd/5608

This Thesis is protected by copyright and/or related rights. It has been brought to you by the The Research Repository @ WVU with permission from the rights-holder(s). You are free to use this Thesis in any way that is permitted by the copyright and related rights legislation that applies to your use. For other uses you must obtain permission from the rights-holder(s) directly, unless additional rights are indicated by a Creative Commons license in the record and/ or on the work itself. This Thesis has been accepted for inclusion in WVU Graduate Theses, Dissertations, and Problem Reports collection by an authorized administrator of The Research Repository @ WVU. For more information, please contact researchrepository@mail.wvu.edu. 


\title{
A Bayesian Approach to Reduced Order Modeling in Catalytic Steam Reforming
}

\author{
Evan D. Ford \\ Thesis submitted \\ to the Benjamin M. Statler College of Engineering and Mineral \\ Resources \\ at West Virginia University
}

in partial fulfillment of the requirements for the degree of

Master of Science in

Mechanical Engineering

David Mebane, Ph.D.,Chair

Fernando Lima, Ph.D.

David Klinke, Ph.D.

Department of Mechanical and Aerospace Engineering

Morgantown, West Virginia

Keywords: Methane Reforming, Bayesian Statistics

Copyright 2015 Evan D. Ford 


\section{Abstract}

An investigation was conducted to determine the feasibility of developing a reduced order model capable of accurately predicting the behavior of steam methane reforming. An emerging model reduction technique based on examining causal relationships was applied to the reaction network developed by $\mathrm{Xu}$ and Froment to eliminate unnecessary intermediate species[1]. A dynamic discrepancy term was included in the reduced network to quantify the error incurred from the network reduction. This discrepancy is stochastic in nature, and a Markov Chain Monte Carlo (MCMC) sampling routine coupled with Bayesian statistical methods was used to calibrate the parameters of the discrepancy by comparison with simulated data provided by a more robust model of methane reforming.

An output distribution of discrepancy parameters was calibrated based on the transient response of a laboratory scale continuous stirred tank reactor (CSTR). Extrapolation to predictions of more complex plug flow reactor (PFR) models was also shown effective using the calculated distribution. Specifications regarding reactor geometry and operating conditions were taken from previously published studies. Traditional simplifying assumptions were specified to reduce the computation complexity of both the reactor simulation and calibration routine. Simulations were performed using a combination of MATLAB ${ }^{\mathrm{TM}}$ and C++ MATLAB ${ }^{\mathrm{TM}}$ executable (MEX) files using high performance computing resources available through West Virginia University's Spruce Knob cluster.

Results of the calibration showed that the proposed modeling technique is able to reproduce the behavior of both the transient response of the single constant stirred tank reactor and the discretized plug flow reactor approximations. Convergence of the calibration routine was validated through statistical means. Additionally, computational times for both the robust model and the proposed reduced model are shown to be on the same order of magnitude. The combination of these findings verifies the ability of the proposed modeling technique to not only accurately predict the behavior of steam reforming but also indicates the potential for applying the proposed method for more complex simulations. 


\section{Contents}

1 Abstract ii

2 Background $\quad 1$

3 Model Development 13

3.1 High Fidelity Model . . . . . . . . . . . . . . . . . . 14

3.2 Reduced Order Model . . . . . . . . . . . . . . . . . . 18

3.2.1 Model Reduction . . . . . . . . . . . . . . . . . 18

3.2.2 Model Kinetics and Dynamic Discrepancy . . . . . . . . . . . . . 23

3.2.3 Bayesian Calibration .................... 26

4 Simulation Methodology 28

5 Results and Discussion $\quad 32$

5.1 Introduction . . . . . . . . . . . . . . . . . 32

5.2 Lab Scale CSTR . . . . . . . . . . . . . . . . . . . . 32

5.3 Convergence Determination . . . . . . . . . . . . . 36

5.4 Lab Scale PFR . . . . . . . . . . . . . . . . . . . . 40

5.5 Industrial Scale PFR . . . . . . . . . . . . . . . . . . 43

5.6 Impact of Discrepancy Terms . . . . . . . . . . . . . . . . . 46

5.7 Temporal Analysis . . . . . . . . . . . . . . . . . . . 48

6 Conclusions $\quad 50$

7 Acknowledgements $\quad 51$ 


\section{List of Figures}

1 Percentage of Hydrogen Production by Process . . . . . . . . . . . . . . . . . 2

2 Demonstration of Equilibrium Limitation for $\mathrm{SMR}$ at $\mathrm{T}=1180^{\circ} \mathrm{F} \ldots \ldots$

$3 \mathrm{Xu} /$ Froment Proposed Reaction Scheme [1] . . . . . . . . . . . . 6

4 Idealized System Full Reaction Network . . . . . . . . . . . . . . . 20

$5 \quad$ Idealized System Reduced Reaction Network . . . . . . . . . . . . . . 21

$6 \quad \mathrm{Xu}$ and Froment Full Reaction Network . . . . . . . . . . . . . . . . . . 22

$7 \quad \mathrm{Xu}$ and Froment Reduced Reaction Network . . . . . . . . . . . . . . 22

8 Example set of Basis Function . . . . . . . . . . . . . . . 25

$9 \quad$ Simulated Reactor Environment . . . . . . . . . . . . . . . . . . . . . 29

10 CSTR Approximation of PFR . . . . . . . . . . . . . . . . 31

11 CSTR Reality Data vs. ROM Realizations . . . . . . . . . . . . . 35

12 Likelihood Calculation for MCMC Sampling . . . . . . . . . . . . . . . 36

13 Bivariate Scatter for $\theta$ Posterior Distributions . . . . . . . . . . . . 38

14 Lab Scale PFR Reality Data vs. ROM Realizations . . . . . . . . . . . . . 42

15 Industrial Scale PFR Reality Data vs. ROM Realizations . . . . . . . . . . . 45

16 ROM CSTR Transient Response without Discrepancy . . . . . . . . . . . . 47 


\section{List of Tables}

1 Summation of Power Law Research . . . . . . . . . . . . . . . . . . . . . 10

2 CSTR Specifications and Initial Conditions . . . . . . . . . . . . . . . 33

3 Convergence Testing Conditions . . . . . . . . . . . . . . . . . 39

4 Convergence Testing Conditions . . . . . . . . . . . . . . . . . 40

$5 \quad$ Lab Scale PFR Specifications and Initial Conditions . . . . . . . . . . . . . 41

6 Industrial Scale PFR Specifications and Initial Conditions . . . . . . . . . 43

$7 \quad$ Simulation Times for Reactor Trials . . . . . . . . . . . . . . . . . . . 48 


\section{Background}

One of the greatest problems facing society is meeting the ever increasing demands for energy. Primarily, petroleum and other fossil fuels have provided this necessary commodity, but their increased use since the Industrial Revolution has lead to a drastic rise in the overall presence of $\mathrm{CO}_{2}$ in the atmosphere[2]. The negative of impact this and other greenhouse gas byproducts of fossil fuel oxidation on the environment has created the need to develop new energy technologies that can both maximize the utility of the remaining deposits of conventional fuels while limiting these repercussions. One of the more promising solutions to this problem is the utilization of hydrogen gas as an energy source due to its higher heating values and nearly emission free combustion[3]. Hydrogen gas already has applications across a wide array of industries including chemicals, food processing, refining industries, and pharmaceuticals, but employing hydrogen as a fuel source is still an emerging technology[4].

Hydrogen gas is utilized in the energy sector as a feed to stacks of fuel cells. These cells use inlet streams of hydrogen and oxygen in the presence of an anode and cathode to produce a flow of free electrons during the formation of water. Fuel cells have practical applications in numerous facets of the energy sector. Low temperature fuel cells have been proven effective in the development of hybrid electric vehicles. Higher temperature fuel cells, such as solid oxide fuel cells (SOFCs), are traditionally used for stationary power generation. Creating electric power and heat from chemical energy, SOFCs have been shown to operate more efficiently than current engines and turbines with a theoretical overall system efficiency of $70 \%$. SOFCs are also favored for producing less pollutants and noise than current counterparts[5]. The vast amounts of hydrogen that exists in nature gives the SOFC great potential as a power source, but the rarity of pure hydrogen is a hindrance, requiring additional refinement from more complex compounds. Commercially, it is more profitable to mass produce hydrogen from methane and other hydrocarbons given the high electricity costs associated with electrolysis[6, 7]. Figure 1 from Ewan \& Allen shows that of all the processes used to refine hydrocarbons, steam methane reformation (SMR) is most widely 
used to meet the current demands primarily due to its cost effectiveness $[3,6]$.

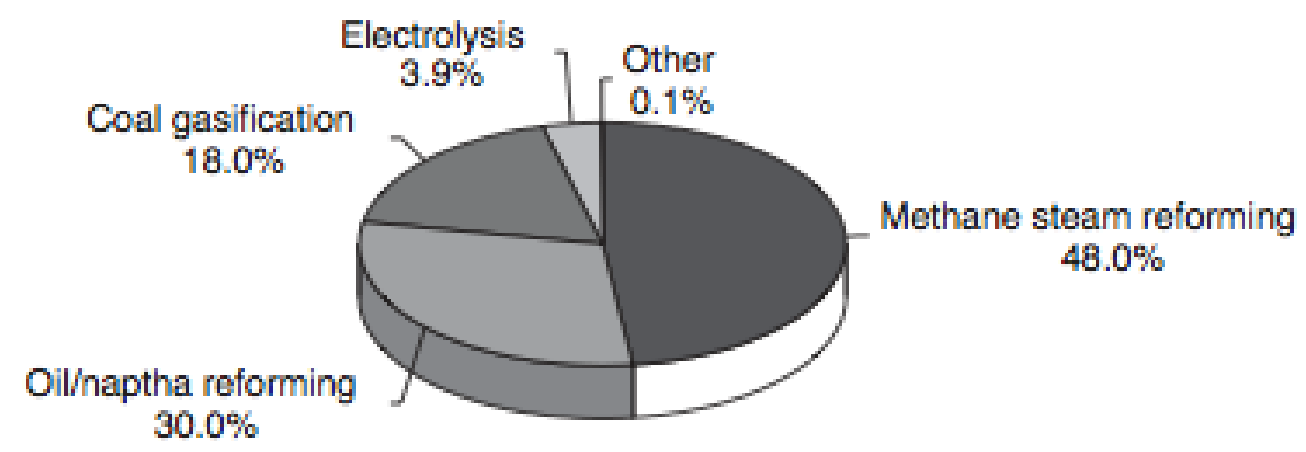

Figure 1: Percentage of Hydrogen Production by Process

SMR is often preferred over other refining process such as partial oxidation and auto reforming because of its high thermal efficiency and higher ratio of $\mathrm{H}_{2}$ to $\mathrm{CO}_{2}$ concentrations $[3$, $8,9]$. Currently, over $75 \%$ of all industrial steam reforming uses natural gas as feedstock. [8] This is because of methane's high hydrogen content and low initial cost as compared to other sources such as coal[3]. This need for new, clean energy sources and the industrial development of the natural gas rich Marcellus Shale region in the Appalachian Basin has led to research into using a methane reforming configured inline with an SOFC to provide a constant inlet hydrogen stream. Steam reforming, given its high thermal efficiency and increased performance at higher temperatures, is ideal given that current SOFC technology requires a high operating temperature $\left(700^{\circ} \mathrm{C}-1000^{\circ} \mathrm{C}\right)[9]$.

Investigations into an SOFC with internal reforming of methane has shown the potential benefit of this inline configuration. The high operating temperature of the SOFC removes the need for additional heat input to the reformer.[10] Sangtongkitcharoean compared the performance of SOFCs operating with both external and internal reforming showing that direct internal reforming was preferred for its ability to operate at lower values of $\frac{F_{\mathrm{H}_{2} \mathrm{O}}}{\mathrm{F}_{\mathrm{CH}_{4}}}[11]$. Wang, et. al. examined the impact the operating parameters has on the local temperature 
and methane conversion for internal reforming, and showed how the heat input from the SOFC boosts performance of the reformer along the length of the reactor[9]. One of the major drawbacks of this configuration is the potential buildup of carbon on the SOFC anode hindering the overall performance $[9,11,12]$. The deposition of carbon and other carbonaceous compounds was found to be most prevalent at the leading edge of the anode, but can be mitigated through higher operating temperature[9, 11]. The potential benefit of this inline configuration behooves the use of process optimization to develop a more pure hydrogen stream from the reformer while minimizing the additional thermal costs necessary to prevent carbon buildup within the SOFC.

In order to produce the necessary hydrogen stream, modifications can be made either to the reactor system to improve conversion at given operating conditions or optimizing the operating conditions impacting the kinetics of the reaction to achieve higher $H_{2}$ production. More often than not, the former of these is preferred primarily due to the ease of implementation since there is still much to learn about kinetics. One promising method for achieving this is in inclusion of a palladium based or other hydrogen permeable membranes to separate hydrogen from the resultant gas mixture[13, 14, 15, 16]. Investigations have shown that the use of the membrane creates a greater conversion of methane and faster production of hydrogen $[13,14]$. However, the additional cost associated with preparation of the membrane hinders this method's implementation on an industrial scale. Other treatment methods to increase hydrogen production often include the addition of either an oxygen secondary reformer or a pre-reformer into the overall reactor design. A secondary oxygen reformer combusts unused methane allowing for more feed gas to be processed without a loss to product purity. This configuration does require additional downstream treatments to handle the greater influx of $\mathrm{CO}_{2}$ produced. A pre-reformer allows for a portion of the hydrocarbon to be reformed before entering the main reactor, and is the product of the development of high activity catalysts[17]. These are able to achieve reforming at lower temperatures and have been shown to be effective at increasing overall hydrogen production 
by Bavarsad[17, 5]. To be effective though, this high activity catalyst requires a much higher nickel content and special treatment to have higher surface area[17].

Another key area of interest that is generally disregarded in the optimization of SMR are the reaction kinetics. The earliest studies into the kinetics of SMR date back to the 18th century, but was not thoroughly investigated until the 1950's[18, 19]. Van Hook provides a thorough summation of the early investigations of SMR (1933-1952) including some introductory research into the governing kinetics[20]. The work of Akers and Camp provides one of the earliest comprehensive investigations into SMR. Their work examined the kinetics over a nickel catalyst for a range of temperatures and pressures. Experimental testing along with a thermodynamic analysis of a proposed network of gas phase reactions showed that the reaction is first order with respect to methane, and that carbon monoxide and carbon dioxide are primary products with carbon dioxide being produced at a much faster pace. Additionally, it was shown that the overall process is equilibrium limited as demonstrated in Figure 2, so additional conditioning is often required for high conversion to hydrogen[21].

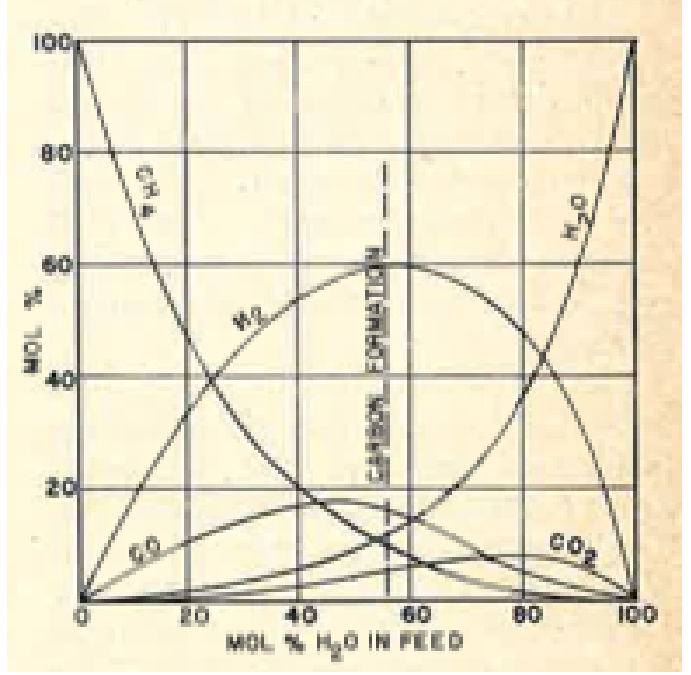

Figure 2: Demonstration of Equilibrium Limitation for $\mathrm{SMR}$ at $\mathrm{T}=1180^{\circ} \mathrm{F}$

Ross and Steel investigated the variability of the catalyst and its impact on the reaction kinetics. Through systematic variations of the catalyst composition, data was collected on the impact on the kinetics and stoichiometry under these slighter alterations[22]. Münster 
and Grabke expanded on this examining SMR on various iron, nickel and iron-nickel alloy catalysts. A flow reactor operating under standard conditions was used to develop a functional relation between the reaction rates and catalyst activity. Additionally, deactivation of the catalyst due to carbon deposits and its impact on kinetics was also alluded to[23].

However, the work of $\mathrm{Xu}$ and Froment has proven to be one of the more thorough studies into steam reforming, and has become the basis for much of the modern research on the subject[24]. The attention paid to the kinetics and their importance in the reforming process is the main distinction separating this work from predecessors. Until this point, $\mathrm{Xu}$ and Froment believed that the value of kinetics in proposed simulations is either neglected or gravely over simplified[18]. Starting with a set of eleven possible reactions involving the main gas phase constituents, a thermodynamic analysis was conducted to eliminate all those incapable of propagating the reaction leaving three main gas phase reactions (GPR):

$$
\begin{array}{llrl}
I & \mathrm{CH}_{4}+\mathrm{H}_{2} \mathrm{O}=\mathrm{CO}+3 \mathrm{H}_{2} & \Delta H=206.1 \mathrm{~kJ} / \mathrm{mol} \\
\mathrm{II} & \mathrm{H}_{2} \mathrm{O}+\mathrm{CO}=\mathrm{CO}_{2}+\mathrm{H}_{2} & \Delta H=-41.15 \mathrm{~kJ} / \mathrm{mol} \\
\mathrm{III} & \mathrm{CH}_{4}+2 \mathrm{H}_{2} \mathrm{O}=\mathrm{CO}_{2}+4 \mathrm{H}_{2} & \Delta H=165.0 \mathrm{~kJ} / \mathrm{mol}
\end{array}
$$

Reaction schemes were then developed based on these GPR to more completely detail how the process advances from reactants to products in the presence of a catalyst. Several guidelines based on literature findings were adhered to for the creation of the differing schemes. These six guidelines govern the interaction of steam and methane with the catalyst and the behavior of the interaction between the radicals:[1]

1. $\mathrm{H}_{2} \mathrm{O}$ reacts with surface atoms, yielding adsorbed oxygen and gaseous hydrogen

2. Methane is adsorbed on surface atoms. The adsorbed methane either reacts with the adsorbed oxygen or is dissociated to form chemisorbed radicals, such as $\mathrm{CH}_{3}^{*}, \mathrm{CH}_{2}^{*}$, $C H^{*}$, and $C^{*}$ 
3. The concentrations of the carbon-containing radicals, $\mathrm{CH}_{3}^{*}, \mathrm{CH}_{2}^{*}, \mathrm{CH}^{*}, \mathrm{C}^{*}$, are much lower than the total concentration of the active sites.

4. The adsorbed oxygen and the carbon containing radicals react to form $\mathrm{CH}_{2} \mathrm{O}^{*}, \mathrm{CHO}^{*}$, $\mathrm{CO}^{*}, \mathrm{CO}_{2}^{*}$

5. The hydrogen formed is directly desorbed into the gas phase and/or the gaseous hydrogen is in equilibrium with $H^{*}$ or $H_{2}^{*}$

6. Each reaction scheme has one "rate determining" step relating to equations I, II, and III with a rate potentially slower than all others, so that it controls the overall reaction.

Following these guidelines a reaction scheme was proposed allowing for parallel formation of $\mathrm{CO}$ and $\mathrm{CO}_{2}$ out of the adsorbed radical $\mathrm{CHO}^{*}$ shown in Figure 3. This scheme equates to the 13 reaction mechanism of elemental steps listed in Equations 1-13.[1]

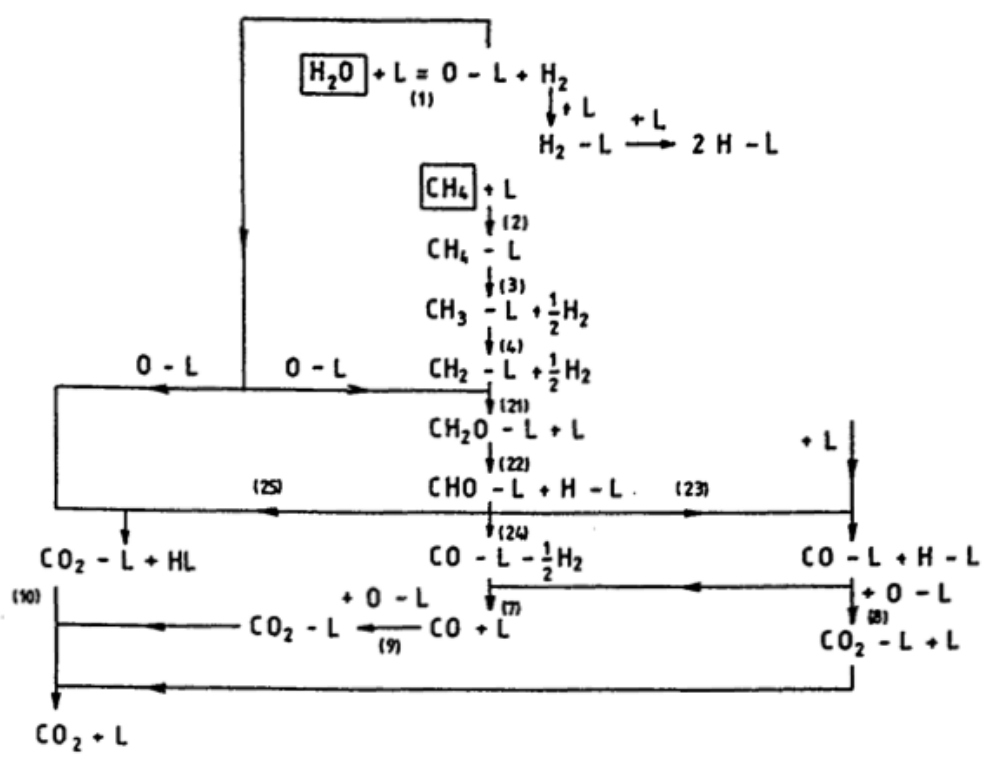

Figure 3: Xu/Froment Proposed Reaction Scheme [1] 


$$
\begin{aligned}
& \mathrm{H}_{2} \mathrm{O}+\mathrm{L}=\mathrm{O}^{*}+\mathrm{H}_{2} \\
& \mathrm{CH}_{4}+\mathrm{L}=\mathrm{CH}_{4}^{*} \\
& C H_{4}^{*}+L=C H_{3}^{*}+H^{*} \\
& \mathrm{CH}_{3}^{*}+\mathrm{L}=\mathrm{CH}_{2}^{*}+\mathrm{H}^{*} \\
& \mathrm{CH}_{2}^{*}+\mathrm{O}^{*}=\mathrm{CH}_{2} \mathrm{O}^{*}+\mathrm{L} \\
& \mathrm{CH}_{2} \mathrm{O}^{*}+\mathrm{L}=\mathrm{CHO}^{*}+\mathrm{H}^{*} \\
& C H O^{*}+L=C O^{*}+H^{*} \\
& C O^{*}+O^{*}=C O_{2}^{*}+L \\
& \mathrm{CHO}^{*}+\mathrm{O}^{*}=\mathrm{CO}_{2}^{*}+\mathrm{H}^{*} \\
& C O^{*}=C O+L \\
& \mathrm{CO}_{2}^{*}=\mathrm{CO}_{2}+\mathrm{L} \\
& 2 H^{*}=H_{2}^{*}+L \\
& H_{2}^{*}=H_{2}+L
\end{aligned}
$$

This kinetic network has been one of the most extensively applied to simulate industrial reactors operating under normal operating conditions. This is due to the generality of the framework which leads itself to greater reliability in results. Also, it helped to resolve contradictions surrounding the reaction order with respect to steam[25]. Additionally, the work of Ding and Alpay showed that this network is suitable for predicting transient response making it one of the few models capable for use in process design[18]. This limit in the number of transient capable networks has slowed the development of optimization algorithms, but advances in high performance computing gives potential for the use of more powerful solving methods to address the problem. Achieving process design and optimization through simulation would allow for the production of predictions without the complications associated 
with physical testing, especially more intricate systems including inline configurations or separation membranes.

Simulation grants the flexibility of application to an large number of reactor designs operating at an infinite set of conditions, but variability of the kinetic mechanism and intense computational needs has hindered its use for $\operatorname{SMR}[3,19]$. Both the value of associated kinetic parameters as well as the overall structure of the kinetic model are highly sensitive to the composition of the catalyst[22]. This makes it impossible to develop a generalized set of equations applicable to all catalyst types and compositions through a simple change in variables[19]. Because of this, a bevy of mechanisms have been created to simulate SMR across a wide range of temperatures $\left(260-1000^{\circ} \mathrm{C}\right)$ and pressures $(100-5000 \mathrm{kPa})[20,25]$. Resolving this kinetics issue would greatly simplify the problem, optimizing SMR and focusing attention to a smaller set of mechanisms.

The other main drawback with the simulation of SMR is the high computational demands required to produce an accurate, realistic simulation. Methane reformation is the combination of a catalytic reactor, fluid dynamics, and heat transfer problem. Trying to incorporate all of this into a single model create a simulation requiring an execution time in excess of what would be profitable. This is often mitigated by a lumped parameter based modeling method which averages key variables in exchange for reduced computational demands as compared to the more complex surface based modeling schemes. These lumped parameter based simulations are better suited for investigations into process and reactor design optimization because of this reduced execution time and that the volume averaged approximation is sufficient for the desired output[3].

Through the use of improved analytic methods, it is desired to advance existing simulation methods to improve overall quality of results while maintaining or improving the computational efficiency. Traditional modeling methods for catalytic reactions are generally built using the Langmuir Hinshelwood kinetics. In this method, the kinetics are based on establishing a rate determining step which dictates the progression of the overall reaction; 
however, application of this to SMR is not as straight forward as would be hoped. Munster's work showed that this rate determining step tends to change depending on the percentage of nickel in the catalyst[23]. Xu and Froment addressed this issue by selecting a rate determining step for each of the gas phase reactions and then building the reaction rates shown in Equations 14-16[1]

$$
\begin{aligned}
& r_{1}=\frac{k_{1}}{P_{H_{2}}^{2.5}}\left(P_{\mathrm{CH}_{4}} P_{\mathrm{H}_{2} \mathrm{O}}-\frac{P_{\mathrm{H}_{2}}^{3} P_{C O}}{K 1}\right) /\left(D E N^{2}\right) \\
& r_{2}=\frac{k_{2}}{P_{\mathrm{H}_{2}}}\left(P_{C O} P_{\mathrm{H}_{2} \mathrm{O}}-\frac{P_{\mathrm{H}_{2}} P_{C O}}{K 2}\right) /\left(D E N^{2}\right) \\
& r_{3}=\frac{k_{3}}{P_{\mathrm{H}_{2}}^{3.5}}\left(P_{\mathrm{CH}_{4}} P_{\mathrm{H}_{2} \mathrm{O}}^{2}-\frac{P_{\mathrm{H}_{2}}^{4} P_{\mathrm{CO}_{2}}}{K 3}\right) /\left(D E N^{2}\right) \\
& D E N=1+K_{C O} P_{C O}+K_{\mathrm{H}_{2}} P_{\mathrm{H}_{2}}+K_{\mathrm{CH}_{4}} P_{\mathrm{CH}_{4}}+\frac{K_{\mathrm{H}_{2} \mathrm{O}} P_{\mathrm{H}_{2} \mathrm{O}}}{P_{\mathrm{H}_{2}}}
\end{aligned}
$$

The accuracy of this model was displayed in reproducing the results of DeDeken's study examining the behavior of a industrial sized reformer while taking into account diffusion limitations[26].

Modern modeling methods use experimental data to develop more precise rate expressions by fitting a generic power law form. Power law fitting can develop an equation for the depletion of methane as a function of the partial pressures each to an initially unknown exponent given by Equation 18. Mogensen, et. al. provides a through investigation of several power law fittings for SMR which are summarized in Table 1 [27].

$$
-r_{\mathrm{CH}_{4}}=k P_{\mathrm{CH}_{4}}^{\alpha} P_{\mathrm{H}_{2} \mathrm{O}}^{\beta} P_{\mathrm{H}_{2}}^{\gamma} P_{\mathrm{CO}}^{\delta} P_{\mathrm{CO}}^{\lambda}
$$


Table 1: Summation of Power Law Research

\begin{tabular}{|c|c|c|c||}
\hline Expression & Support & $\mathrm{T}\left({ }^{\circ} \mathrm{C}\right)$ & Ref. \\
\hline$k P_{C H_{4}}^{1.19}$ & $\mathrm{CGO}$ & $800-950$ & {$[28]$} \\
\hline$k P_{C H_{4}}^{0.85} P_{H_{2} O}^{-0.35}$ & $\mathrm{YSZ}$ & $850-950$ & {$[27]$} \\
\hline$k P_{\mathrm{CH}_{4}} P_{\mathrm{H}_{2} \mathrm{O}}^{-1.28}$ & $\mathrm{ZrO} 2$ & $800-1000$ & {$[29]$} \\
\hline$k P_{C H_{4}}^{1.20}$ & $\mathrm{ZrO} 2$ & $900-1000$ & {$[30]$} \\
\hline$k P_{C H_{4}}^{1.3} P_{\mathrm{H}_{2} \mathrm{O}}^{-1.2}$ & $\mathrm{YSZ}$ & - & {$[31]$} \\
\hline
\end{tabular}

This method has the advantage of being able to create a expression for the rate of reaction without the need for a mechanism of elementary steps. However, many of the studies that apply this fitting do not follow the recommended procedure for measuring catalytic reaction rates set by "Concepts of Modern Catalysis and Kinetics", and often fail to take into account the effects of mass transport. Furthermore, power law fitting is very specific to the system being measured, so extrapolating the rate law to various model configurations is highly problematic[27].

Advancements in high performance computing have allowed for more modern methods to address the current issues regarding simulating SMR. Some of the way this has been attempted involve addressing the problem in varying contexts, and through the use of more modern computational algorithms. Making analogies between and other engineering concepts can allow for new, advanced methods for addressing the issues for modeling SMR created by traditional means. These unique comparisons allow for the application of simplifications not valid to standard reactor problems. Mokheimer, et. al. applied this concept to create a highly accurate model for SMR by applying the rates in Equations 14-16 in the context of a computational fluid dynamics (CFD) problem. It was desired to create a new solution method with results accuracy equal to the results of traditional means while reducing the necessary computational time. Model validity up to $99.75 \%$ was noted with this when comparing this CFD analysis to the results of Xu and Froment's simulations; however, 
no mention was made to the computing efficiency of the fluid dynamics approach[3].

Another example of one of these cross discipline analogies is demonstrated by Liu in his thesis to relate rector optimization with electrical engineering[32]. Investigating carbon buildup of SOFC anodes due to impurities of internal reforming, an adjusted form of the reaction network proposed by $\mathrm{Xu}$ and Froment was equated to a comparable resistor network allowing for the use of Kirchhoff laws and loop analysis to analyze the overall system. Resistances for the network were associated with the rates of the elementary steps of the Xu and Froment reaction scheme determined using a microkinetic study. Simplifications were made by combining resistors in series and parallel to remove the unnecessary steps from the system and creating a streamlined reduced model. The use of the resistor network allowed for much faster simulation speeds, but the produced results showed a large error in terms of methane conversion when compared to previous results that could not be explained[32]. So currently, this approach to SMR modeling still needs improvements in order to produce a valid simulation that is also time and computationally more efficient.

The more conventional way of tackling the issues with modeling SMR applies more sophisticated computing algorithms to solve the proposed system of equations. The main issue that arises is quantifying the uncertainty associated with the complexity of the solution methodology. Fine tuning by calibrating key parameters is one such way of reducing this problem. Sciazko et. al. apply this idea using a generalized least squares method to determine key parameters and unknown values. The main difference separating the generalized least squares from the traditional application of least squares is that it allows for the number of equations to be different than the amount of measured values[10]. This allows for a more general investigation to provide accurate results that include the uncertainty[33]. In this problem; however, because the constraint equations in this application are differential they need to be linearized to be applicable to the solution method. This greatly limits the region in which the calibration can converge to an accurate solution, requiring additional work in selecting the initial values for the known and unknown parameters. Ideally, an improvement 
on this would be able to yield the high accuracy of result without the need for such precision on the initial guess of the associated parameters.

The proposed reduced order modeling methodology attempts to address the current issues associated with computational simulation of SMR while improving upon the methods presented. A new model for SMR simulation has been developed using a non-parametric regression technique incorporating a Bayesian smoothing spline ANOVA (BSS-ANOVA) procedure for variable and search direction selection[34]. This model is the product of applying dynamic reduction methodology to the reaction network in Equations 1-13 to minimize the total number of necessary parameters required to create a valid simulation. These simplifications induce additional uncertainty driving the model further from the reality. Including the dynamic discrepancy function helps to quantify this and allow for the reduced model to accurately represent the high fidelity model. A multivariate normal distribution is assumed for the parameter set and calibration is performed through comparison with data to determine the output distribution. Search direction sampling via a Markov Chain Monte Carlo routine is used to search for variable sets that are able to match the data. Developing the model in this way allows for the decomposition of the variable selection into main effects and secondary effects allowing for the study of each of their contributions to the model fit. In addition the orthogonality of the ANOVA framework ensures that there is no overlap in contribution from corresponding primary and secondary effects[34].

BSS-ANOVA has shown promising results when compared with other similar modeling schemes. Reich, et. al. compare the BSS-ANOVA with a multivariate adaptive regression splines (MARS), component selection and smoothing operator (COSSO) and a Gaussian process model to determine the quality of results and necessary computational time for each using a "toy" modeling problem. Their work showed that for varying designs of the same simulation, the BSS-ANOVA technique was able to reproduce on par or superior curves to its competitors for each trial[34]. Storlie also used this method to reproduce the results of a fluid dynamics problem initially performed by Gaussian process models for simulation anal- 
ysis. Results of the study showed that the BSS-ANOVA methodology provided an equal or greater average posterior probability, and in one instance operated faster than the Gaussian modeling[35]. Other published applications of the BSS-ANOVA routine include a Carbon Capture Simulaiton Initiative (CCSI) study of the uptake of $\mathrm{CO}_{2}$ in amine sorbents[36]. Additionally, current work is exploring the capability of implementation to modeling carbon capture in mesoporous sorbents.

The following report details the development of a simulation model for SMR following the Bayesian method mentioned above. Subsequent chapters detail the development of the necessary computational models from first principles including the reduction of a robust reaction network and the implementation and calibration of the dynamic discrepancy terms; the methodology used to collect data for various flow reactors of both the lab and industrial scale; and the presentation of results from the reactors with discussion about the feasibility of application.

\section{Model Development}

Demonstrating the proposed method requires the development of two unique, simulation models predicting the behavior of methane reforming. The first of these, designated the high fidelity model (HFM), is the more robust of the two taking into account all of the gas phase and adsorbed species that $\mathrm{Xu}$ and Froment[1] listed in their reaction network. This serves to create realistic data sets used to compare realizations of the second model, the reduced order model (ROM), to guide the calibration of the included discrepancy terms. The structure of this second model is derived from applying simplification measures to minimize the number of species in the proposed reaction network to theoretically provide an accurate prediction. 


\subsection{High Fidelity Model}

The main priority of the HFM is to provide the necessary calibration data to tune the various parameters included in the reduced model. Normally, this data can either be taken from experimental trials, or the simulation results of a more robust model. For this purpose, the latter was chosen to better demonstrate the power of the proposed methodology. The HFM allows for the synthesizing of data across a variety of operating conditions for both laboratory and industrial sized reactors. It was desired for the HFM to be fairly complex allowing for a much greater reduction in order to determine the ability of the calibration routine to be feasible in terms of computing needs to reproduce realistic results.

Development of the HFM is based on the reaction network proposed by $\mathrm{Xu}$ and Froment (Equations 1-13). The 13 step reaction network details methane reforming using 16 unique chemical species: 5 gas phases, 10 adsorbed radicals, and the available bonding sites on the catalyst. A time dependent balance equation is required for each chemical species within the system leading to a multi-variable ODE system. Since there is a mixture of gas phase and adsorbed species involved, this must be reflected in the corresponding balances to account for the physical behavior of each in the reactor. Gas phases are more intricate given the combination of a flow problem with a catalytic reaction. Generically, the concentration balance for each gas species, $C_{i}$, is expressed by Equation 19 demonstrating the balance of inflow and outflow, $\nu_{0}$ and $\nu$, with the rate of either production or depletion, $r_{i}$.

$$
\frac{d C_{i}}{d t}=\frac{C_{i, 0} \nu_{0}-C_{i} \nu}{V}+r_{i}
$$

Recalling the overall gas phase reactions, it can be seen that the process is not equimolar (moles into system is not equal to moles out), so as the reaction progresses a higher volumetric flow rate from the system is necessary to prevent accumulation as demonstrated in the numerator of the final term of Equation 19. Inert nitrogen is also included to allow for simulating scenarios in which the reactor initiates form a voided state bringing the total 
number of tracked species up to 17.

In a similar fashion, tracking the change of the adsorbed species over time can be expressed in terms of the rate of its production and depletion. A site limited model is employed to normalize the concentration of each species adsorbed to the catalyst surface $\left(S_{i}\right)$ to the total number of bonding $\operatorname{sites}\left(S_{t o t}\right)$ as shown in Equation 20. Additionally, since the catalyst is assumed to be stationary the overall mass balance equation can be simplified to the from given in Equation 21.

$$
\begin{gathered}
X_{i}=\frac{S_{i}}{S_{t o t}} \\
\frac{d X_{i}}{d t}=r_{i}
\end{gathered}
$$

Using the site limited assumption requires the application of a conservation equation to bound the total amount of available sites on the catalyst surface. This is formed by determining the percentage of available catalyst sites from the sum of the site fractions (Equation 22) of all the adsorbed species then differentiating with respect to time resulting in Equation 23.

$$
\begin{aligned}
X_{L} & =1-\sum X_{i} \\
\frac{d X_{L}}{d t} & =-\sum \frac{d X_{i}}{d t}
\end{aligned}
$$

It should be noted that in Equations 19 and 21, the rate of reaction terms presented $\left(r_{i}\right)$ are indicative of the sum of the rates of the elementary steps in which the given species $i$ is produced less the sum of the rates in which it is consumed demonstrated in Equation 24 . The rate for each of these elementary steps takes on the form shown in Equation 25 which demonstrates the equilibrium limiting nature of methane reforming in the parenthetical term incorporating the equilibrium constant. 


$$
\begin{gathered}
r_{i}=\sum r_{j, \text { prod }}-\sum r_{j, \text { cons }} \\
r_{j}=k_{j}\left(\prod P_{j, \text { react }} X_{j, \text { react }}-\frac{\prod P_{j, \text { prod }} X_{j, \text { prod }}}{K_{j}}\right)
\end{gathered}
$$

Lastly, estimating the kinetics parameters of the HFM provides a unique challenge given the limited data available on the adsorbed radicals. As a simplifying assumption, the rate and equilibrium constants, $k_{j}$ and $K_{j}$ respectively, associated with each rate expression were assumed to be on the order of those corresponding to the kinetic values associated with GPR I and III. Values were assigned based on determining which GPR each elementary step could be used to reproduce through combination with other steps in the network. Those that could be used to express multiple GPR were given averaged kinetic values corresponding to an assumed equal selectivity. Base values for these kinetic parameters were estimated using a from of the Arrhenius equation which extrapolates the values at any temperature from a given reference point[1].

$$
k_{i}=k_{i, T_{r e f}} \exp \left[-\frac{E_{i}}{R}\left(\frac{1}{T}-\frac{1}{T_{r e f}}\right)\right] \quad ; \quad i=I, I I I
$$

Because of the low concentration of $\mathrm{CO}$ observed in experimental trials, it is difficult to get a valid estimation of the rate at which GPR II operates leading to the assumption that it constantly near equilibrium[1]. This has the advantage of providing the algebraic expression shown in Equation 27 to incorporate in the model allowing for simplification and reduction in the overall number of ODE's.

$$
K_{2}=\frac{C_{\mathrm{CO}_{2}} C_{\mathrm{H}_{2}}}{C_{\mathrm{CO}} C_{\mathrm{H}_{2} \mathrm{O}}}
$$

Values of the equilibrium constants are taken directly from thermodynamic data with the exception again being GPR II whose equilibrium constant was estimated by Callaghan in her dissertation[37].

$$
K_{i}=\exp \left(\frac{\Delta S^{\circ}}{R}\right) \exp \left(\frac{\Delta H^{\circ}}{R T}\right)
$$




$$
K_{2}=\exp \left(-2.4198+0.0003855 T+\frac{2180.6}{T}\right)
$$

Assimilating the kinetics and the rate expressions into the mass balance Equations 19 and 21 finalizes the 17 reaction network constituting the HFM.

$$
\begin{aligned}
& \frac{d C_{\mathrm{H}_{2} \mathrm{O}}}{d t}=\frac{C_{\mathrm{H}_{2} \mathrm{O}, 0} \nu_{0}-C_{\mathrm{H}_{2} \mathrm{O}} \nu}{V}-r_{1} \\
& \frac{d C_{C H_{4}}}{d t}=\frac{C_{C H_{4}, 0} \nu_{0}-C_{C H_{4}} \nu}{V}-r_{2} \\
& \frac{d C_{C_{2}}}{d t}=\frac{-C_{C O_{2}} \nu}{V}+r_{11} \\
& \frac{d C_{H_{2}}}{d t}=\frac{-C_{H_{2}} \nu}{V}+r_{1}+r_{13} \\
& \frac{d C_{N_{2}}}{d t}=\frac{-C_{N_{2}} \nu}{V} \\
& \frac{d X_{O}}{d t}=r_{1}-r_{5}-r_{8}-r_{9} \\
& \frac{d X_{C H_{4}}}{d t}=r_{2}-r_{3} \\
& \frac{d X_{C H_{3}}}{d t}=r_{3}-r_{4} \\
& \frac{d X_{C H_{2}}}{d t}=r_{4}-r_{5} \\
& \frac{d X_{\mathrm{CH}_{2} \mathrm{O}}}{d t}=r_{5}-r_{6} \\
& \frac{d X_{C H O}}{d t}=r_{6}-r_{7} \\
& \frac{d X_{C O}}{d t}=r_{7}-r_{8}-r_{10} \\
& \frac{d X_{C O_{2}}}{d t}=r_{8}+r_{9}-r_{11} \\
& \frac{d X_{H}}{d t}=r_{3}+r_{4}+r_{6}+r_{7}+r_{9}-2 r_{12} \\
& \frac{d X_{H_{2}}}{d t}=r_{12}-r_{13} \\
& \frac{d X}{d t}=-\sum \frac{d X_{i}}{d t} \\
& C_{C O}=\frac{C_{C O_{2}} C_{H_{2}}}{K_{2} C_{H_{2} O}}
\end{aligned}
$$

A continuous stirred tank reactor (CSTR) was chosen for the modeling environment al- 
lowing the assumptions of well mixed and species molar flow rates proportional to its percent concentration in the reactor. Additionally, an isothermal condition is employed making the assumption of ideal temperature control due to the presence of a heater. MATLAB ${ }^{\mathrm{TM}_{\mathrm{w}}}$ was used for coding the HFM taking advantage of the built in ODE solver and the "ode45" function.

\subsection{Reduced Order Model}

While the HFM is a basic exercise in following the algorithms of reaction engineering and reactor design, the development of the reduced order model is broken down into three steps: parameter selection, kinetic modeling with dynamic discrepancy, and Bayesian calibration. This process allows for the creation of a highly accurate model based on the fewest number of chemical species necessary. Determining this minimum parameter set is achieved through the application of a system reduction technique examining casual relationships to remove all unnecessary intermediate chemical species. Reducing the parameter set minimizes the order of the ODE set, and with it the computational intensity, at the cost of inducing error in the simulation. Dynamic discrepancy is applied to the kinetic parameters to recover some of the variability lost due to the network reductions. This discrepancy uses a set of stochastic coefficients paired with deterministic functions to produce a correctional factor that is then incorporated into the model. Initially, nothing is known about the values of these coefficients, so an overarching Gaussian multi-variate distribution is assumed to cover all possible solution sets. Then Markov Chain driven random walk calibration routine coupled with Bayes' theorem is used to narrow this distribution down to a confined region in parameter space.

\subsubsection{Model Reduction}

Scaling down the number of model parameters of the $\mathrm{Xu} /$ Froment reaction network requires a systematic approach to gauge which chemical species are essential. The dynamic system 
reduction technique suggested by Takens proposes that any system can be reformed in terms of only the necessary variables by adapting the governing equations[38]. To illustrate an example, take the idealized reaction network detailing the creation of species $\mathrm{H}$ from system reactants $\mathrm{A}$ and $\mathrm{D}$ :

$$
\begin{aligned}
& A=B+C \\
& C+D=E+F \\
& B+F=G \\
& E+G=H
\end{aligned}
$$

Takens' theorem shows that the information about any particular time series is passed on to any series that it creates[38]. This allows for the reduction of dynamic networks based on causal relationships without the loss of vital information.

Creating a reaction graph based on the network mechanism allows for quicker identification of the desired causal relationships. Connections between the different species are made through nodes representing the listed chemical reactions. This provides a more manageable way of visualizing how the system progresses from start to finish, and is necessary when determining the species required by the reduced model. Figure 4 shows this concept applied to the idealized system above.

With the network graph in place, intermediate species can be quickly identified. In this case, species B, C, E, F, and G are all intermediate states that have potential for removal. According to Takens' theorem, a minimal set of parameters containing the variability of the full network can be determined through exploiting the various causal relationships. As an example, species $\mathrm{A}, \mathrm{B}$, and $\mathrm{G}$ are related given that $\mathrm{A}$ reacts to create $\mathrm{B}$ which reacts a second time to create $\mathrm{G}$. This indicates that all the information regarding species $\mathrm{B}$ is contained within A, so a relation directly linking A to $\mathrm{G}$ can be created combining the kinetics of two original reactions. This simplification makes species B no longer important 


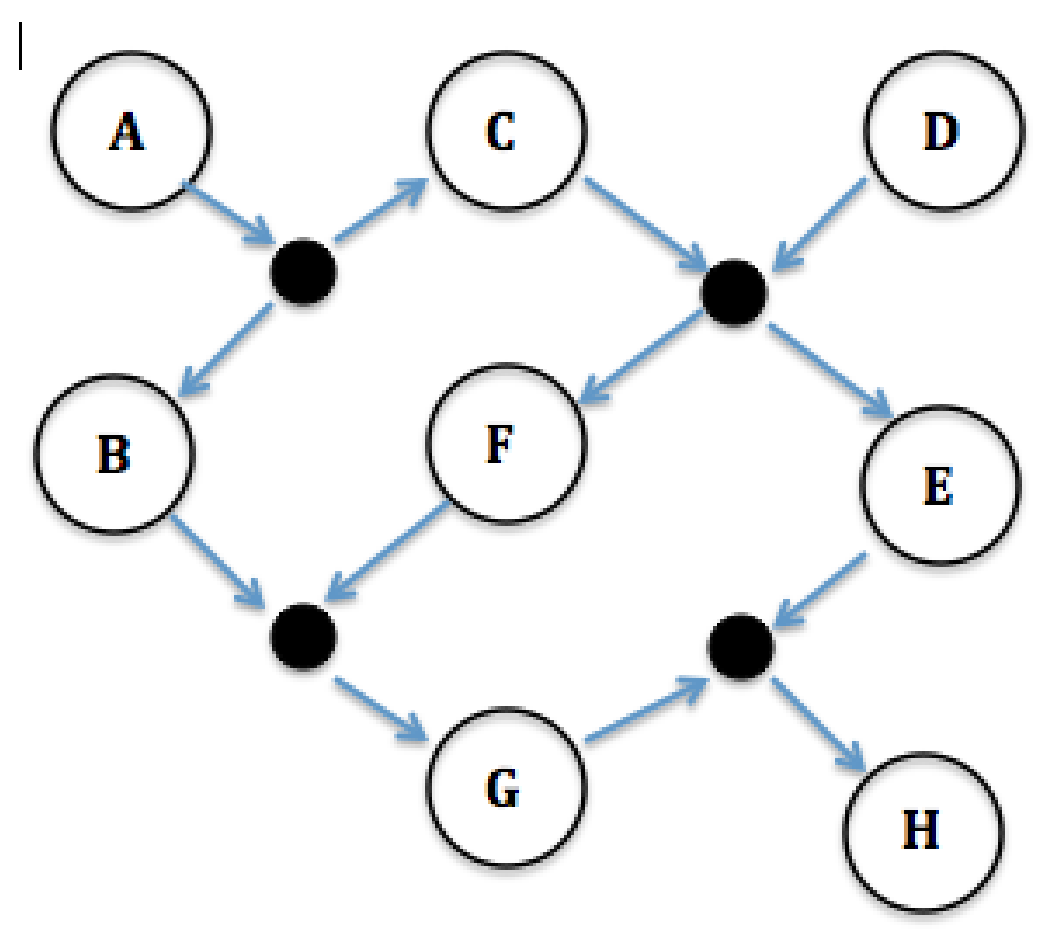

Figure 4: Idealized System Full Reaction Network

to the model, but still maintains the information in its time series. Reductions such as this are done systematically until all intermediate states are removed leaving the streamlined network graph shown in Figure 5.

Making network reductions in this manner maintains full variability associated with the original expanded model. In this case, the information about the intermediate states is contained with in the time series for species A, D, and H. So greater simplifications leads to more information incorporated into fewer variables. The addition of the discrepancy functions works to extract this condensed information allowing the reduced configuration to match the behavior of the higher fidelity network despite the limited number of total variables. 


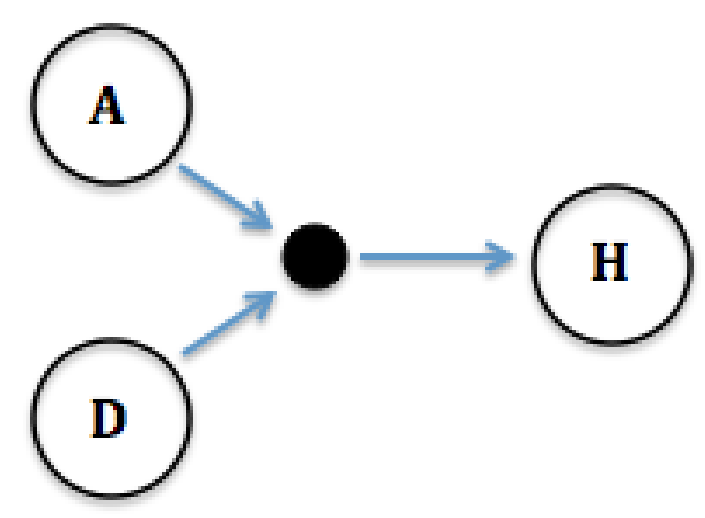

Figure 5: Idealized System Reduced Reaction Network

This model reduction methodology was applied to the $\mathrm{Xu}$ /Froment network to determine the minimum number of essential states to describe SMR and to produce the proposed reduced order model. A complex reaction graph was devised in a manner similar to that of the idealized example. The 16 gas phase and adsorbed species are all related into the mapping of the "flow" of the reaction from reactants to products. Figure 6 depicts this full network graph. The addition of nitrogen is not included in this because it is inert in regard to the chemical reactions./clearpage

By inspection several of the simplifications are clearly presented (ex. relating $\mathrm{CH} 4$ to its adsorbed radicals CH4-L, CH3-L, and CH2-L; gas phase products to their adsorbed counterparts; and removal of $\mathrm{CH} 2 \mathrm{O}-\mathrm{L}$ by node collapsing) while others require careful investigation of the network's structure. Fully simplifying the system leaves a streamlined network dependent on only the gas phases present in steam methane reforming as shown in Figure 7. For this purpose, the presence of the catalyst and its absorbed states are removed from the model, but their information is still contained within the gas species. This removes the need for the site balance equations in the ROM.

The remaining species form a basis around which the kinetics of the ROM are formed. This new kinetic model will have to account for all the information lost due to the removal 


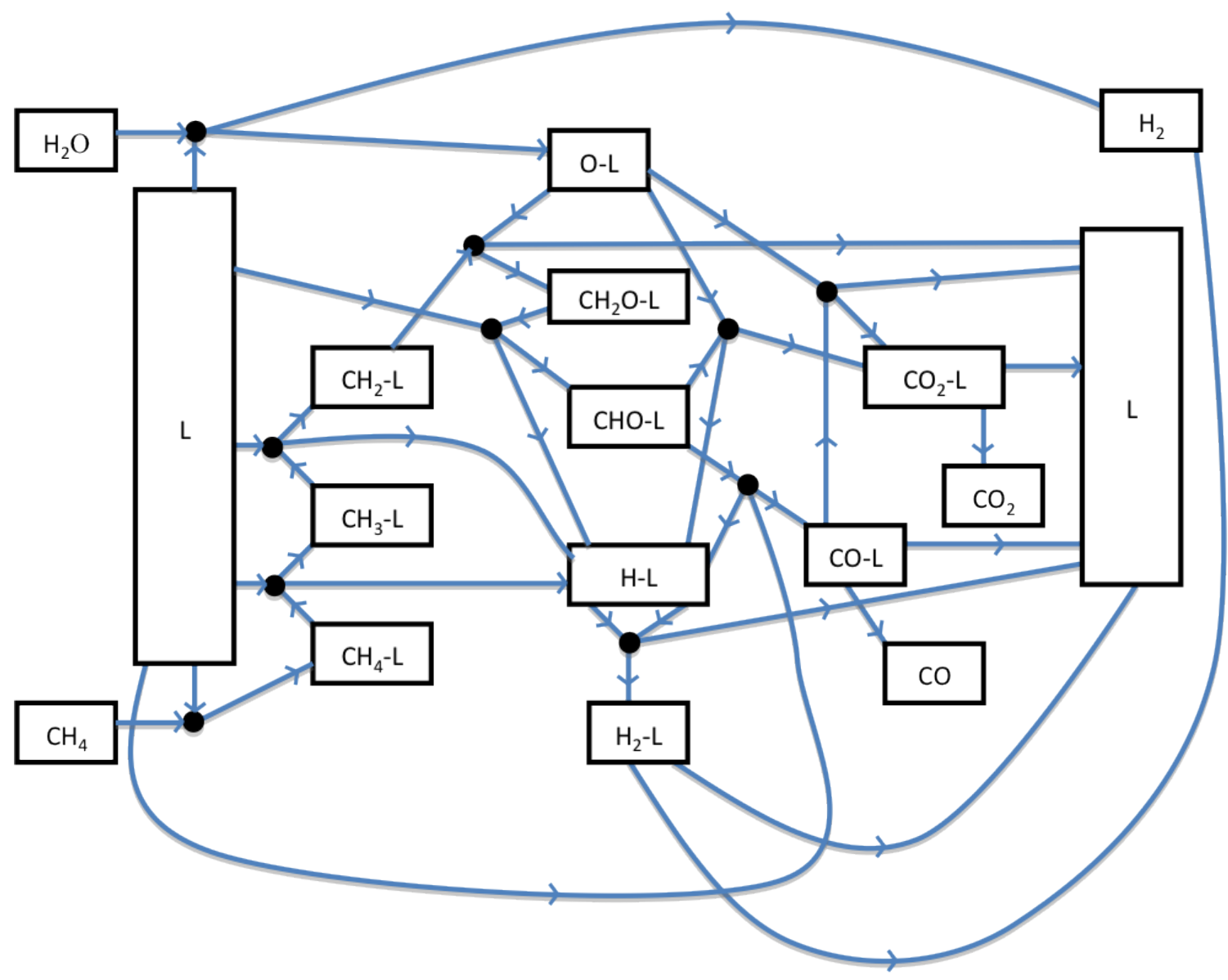

Figure 6: Xu and Froment Full Reaction Network

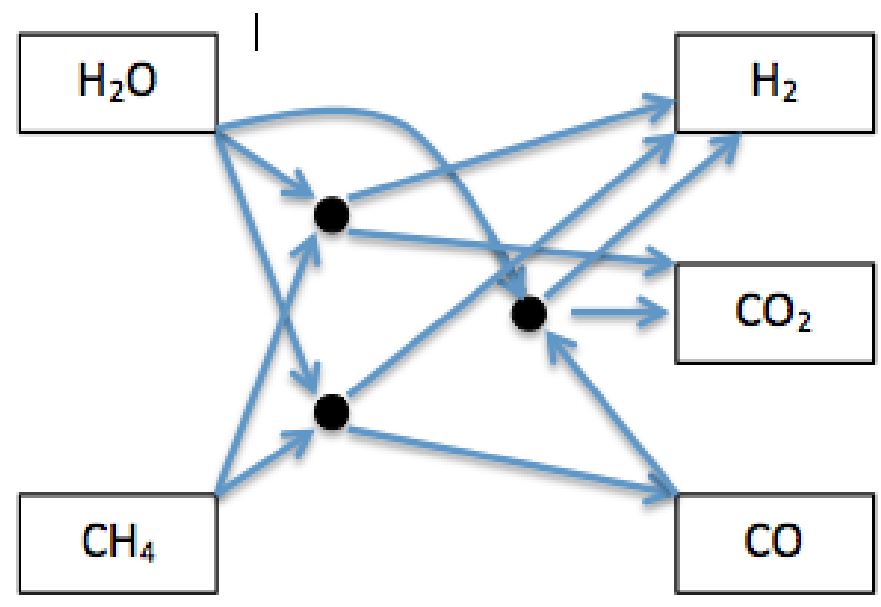

Figure 7: Xu and Froment Reduced Reaction Network

of species as a result of the simplifications. 


\subsubsection{Model Kinetics and Dynamic Discrepancy}

The reduction in variables from 17 to 6 necessitates the development of a new reaction network and kinetic system to better suit the nature of the minimal set of states in the reduced order model. The lack of adsorbed radicals allows for the use of the three GPR (Equations I, II, and III) for this purpose, but additional measures are required to account for the induced error associated with the dynamic model reduction. In many ways, the structure of the reduced model mimics that of the high fidelity model, especially in the general form of the mass balance ODEs and the applied rate expressions. Stoichiometry and variable flow rate are again taken into account to prevent gas accumulation as the reaction occur, as well as the equilibrium condition implied on Equation II. The full set of ODEs calculated in the reduced model is summarized in Equations 47-52.

$$
\begin{aligned}
& \frac{d C_{\mathrm{H}_{2} \mathrm{O}}}{d t}=\frac{C_{\mathrm{H}_{2} \mathrm{O}, 0} \nu_{0}-C_{\mathrm{H}_{2} \mathrm{O}} \nu}{V}-r_{I}-r_{I I I} \\
& \frac{d C_{C H_{4}}}{d t}=\frac{C_{C H_{4}, 0} \nu_{0}-C_{C H_{4}} \nu}{V}-r_{I}-\frac{1}{2} r_{I I I} \\
& \frac{d C_{C O_{2}}}{d t}=\frac{-C_{C O_{2}} \nu}{V}+\frac{1}{2} r_{I I I} \\
& \frac{d C_{H_{2}}}{d t}=\frac{-C_{H_{2}} \nu}{V}+r_{I}+2 r_{I I I} \\
& \frac{d C_{N_{2}}}{d t}=\frac{-C_{N_{2}} \nu}{V} \\
& C_{C O}=\frac{C_{C O_{2}} C_{H_{2}}}{K_{2} C_{H_{2} O}}
\end{aligned}
$$

What distinguishes the ROM is the treatment of the kinetics. While the form of the rate expressions is nearly identical to the HFM (see equation 25) the form of the kinetic values in the ROM are augmented to incorporate the discrepancy functions. Because of the network reduction, the approximations used in the HFM are no longer sufficient to implement in the ROM. Applying dynamic discrepancy bridges the gap in kinetic values between the ROM and HFM created by the model reduction. 
The dynamic discrepancy consists of two parts: a set of deterministic basis functions and a vector of stochastic coefficients. Combined, these are used to quantify the uncertainty allowing the behavior of the reduced model to match that of the high fidelity. The generic form of the discrepancy term is shown in Equation 53. Essentially, this is built piecewise from examining the influence of each state variable independently and then building up to more complex interactions.

$$
\delta=\sum \beta_{i} \phi_{i}(x)+\sum \beta_{i j} \phi_{i}(x) \phi_{j}(y)
$$

Each interaction is comprised of a series of basis functions with a corresponding beta value based on the number of the basis function and the state variable. These basis functions are oscillatory in nature starting at high amplitude and low frequency and decrease amplitude and increase frequency at higher order as demonstrated in Figure 8 taken from [35]. Combining these functions gives the discrepancy potential to take a myriad of shapes, allowing it to adhere to any behavior represented in the realistic data. The main criteria affecting the overall form of Equation 53 is the number of basis functions required for each of the first and second order interactions.

Determining the "correct" number of variable interactions and basis functions per interaction is an exercise of evaluating tradeoffs between accuracy and complexity. Inclusion of higher order interactions (i.e. two variable and three variable) dramatically increases the capabilities of the discrepancy at the cost of higher computational complexity. Often this decision is dictated by the form of the data; more complex curves will require a more intricate discrepancy. Once the highest order of variable interaction is determined, optimizing the number of necessary basis functions per interaction is more of a fine tuning process. Ideally, increasing the number of basis functions used within each discrepancy term should allow for a more accurate quantification of the uncertainty between the reduced model and the realistic data; however, the nature of these functions creates a point of diminishing returns in terms of 

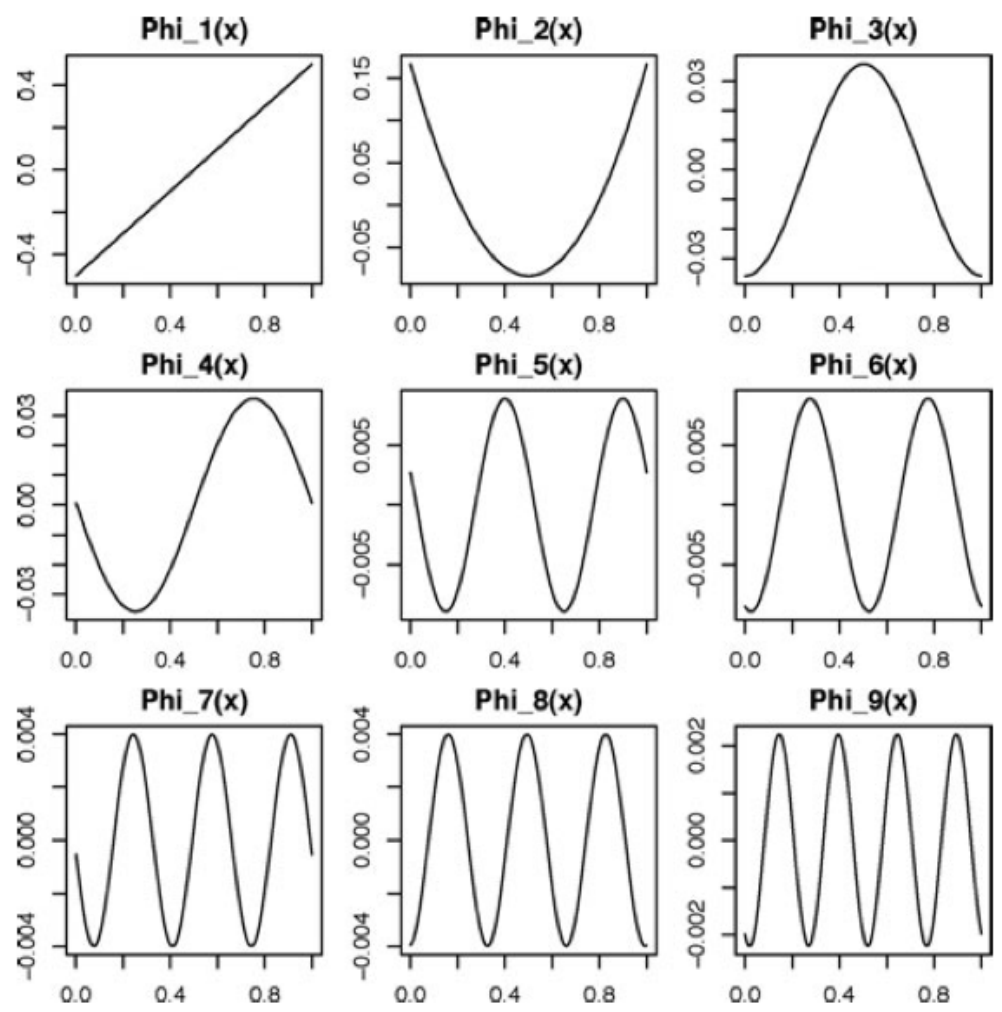

Figure 8: Example set of Basis Function

the additional benefit to the error calculation against the increase in required computational time. Currently there are ways of pinpointing this boundary primarily through examining the beta values for each interaction. After a certain point, the beta values associated with higher order basis functions should trend towards zero, and based on this, truncation can be made on an interaction by interaction basis.

In terms of the ROM, each kinetic parameter has a unique discrepancy term based on the state variables it is functionally dependent on. Computationally, the discrepancy serves as a correctional factor adjusting the base values of the kinetic parameters. First and second order state variable interactions are incorporated in Equation 53 accounting for the presence of each gas phase (first term) as well as the interactions between two unique states (second term). 


$$
\begin{aligned}
k_{i} & =k_{i, 0} \exp \left[\delta_{k_{i}}(x, y ; \vec{\beta})\right] \\
K_{i} & =K_{i, 0} \exp \left[\delta_{K_{i}}(x, y ; \vec{\beta})\right]
\end{aligned}
$$

A total of 92 basis functions with a corresponding beta value are used in each discrepancy term: four first way interactions each with eight functions, and six second order interactions with ten functions. In order to link the computer science with the physical nature of the problem, beta values associated with identical chemical interactions were held consistent across discrepancy terms, but were unique to each type of kinetic parameter. For example, the first order interaction of $\mathrm{H}_{2} \mathrm{O}$ has two sets of values: one set consistent across the discrepancies associated with $k_{1}$ and $k_{3}$, but is uniquely different from the second set which is consistent across the $K_{1}, K_{2}$, and $K_{3}$ discrepancies. Altogether, this equates to a total of 270 beta values (130 for the rate constants and 140 for the equilibrium).

\subsubsection{Bayesian Calibration}

The final step in the production of the ROM is the calibration routine which uses a MCMC to determine the posterior distribution for both the physical and discrepancy parameters. The underlying idea is that there exists a single set of values that, when applied, allows for the reduced model to perfectly match the response of the HFM. This is expressed in the statistical model presented by Kennedy and O'Hagen which suggests that vector comprising the reality data, $\mathrm{Z}$, is a possible realization of the ROM and a combination of three distinct parts: the model $\mathrm{Y}$ and its parameters, $\theta$; the discrepancy $\delta$ and its parameters, $\beta$; and the error, $\epsilon$, and its parameters, $\psi$ as shown in Equation 56.[36] The goal of the calibration is to find a distribution referred to as the posterior.

$$
Z=Y(\theta)+\delta(\beta)+\epsilon(\psi)
$$


This distribution is the result of applying Bayes' theorem given by Equation 57 . The posterior is just one of the three major components constituting Bayes' theorem along with the prior distribution and likelihood.

$$
\Omega(\theta, \beta, \psi \mid Z) \propto \mathcal{L}(Z \mid \theta, \beta, \psi) \pi(\theta, \beta, \psi)
$$

The prior distribution, $\pi(\theta, \beta, \psi)$, gives the initial probability of observing the given set of discrepancy and physical parameters. Prior distributions were assigned to each parameters based on their physical nature within the system and their roll in the statistical model. A multivariate normal distribution is used for the $\beta$ priors to keep the distribution vague and remove any possibility of excluding any solution sets. $\theta$ values are also given a normal distribution, but these are bounded since the values they represent have upper and lower extrema based on physical conditions. Lastly, an inverse gamma distribution is used for the $\epsilon$ since it is best suited for controlling the variance given the uninformative priors associated with $\beta$.

The likelihood, $\mathcal{L}(Z \mid \theta, \beta, \psi)$, gives the probability of observing the realistic data given the current set of discrepancy parameters. All this is normalized by integrating over all possible values associated with the problem. The complexity of the integration necessitates the use of Markov Chain Monte Carlo (MCMC) routine to calculate the desired posterior distribution, $\Omega(\theta, \beta, \psi \mid Z)$.

The MCMC initially draws a sample from the prior distribution which is then passed to Equation 57 to determine the likelihood. The value of the likelihood is used to decide whether the proposed sampling is to be included into the posterior distribution. Based on that value, the draw is either accepted and becomes the next base point for the random walk of the MCMC, or the draw is rejected and a new draw is taken. Increasing the number of MCMC steps helps drive the random walk through parameter space until the routine converges on a confined region, defines the posterior distribution, and finalizes the ROM.

The two models detailed above were used to test the theory that this technique of model 
reduction coupled with the dynamic discrepancy is capable of formulating a suitable ROM to reproduce realistic results for SMR. MATLAB

texttrademark programs were written for both reactors taking advantage of the "ode45" function to solve the ODE systems that constitute the respective process models. Code for the implementation of the reactor models into the Bayesian framework with the MCMC sampling was also done using MATLAB ${ }^{\mathrm{TM}}$, and was developed and distributed by Sham Bhat of the Statistical Sciences group at Los Alamos National Laboratory.

\section{Simulation Methodology}

Simulation trials were carried out to determine the capability of the proposed Bayesian calibration methodology to accurately predict the behavior of methane reforming through both time and spatial propagation of reactor systems. A posterior distribution for the parameters associated with the discrepancy function is calibrated by comparison of the realizations of the ROM for the case of a transient CSTR with its associated HFM. The resulting posterior distributions were then applied to PFR systems on both the lab and industrial scale approximated through several control volumes each with the behavior of the initial CSTR system. The results of the corresponding ROMs and HFMs were again compared to determine if the calibration method could accurately predict the steady-state concentrations of the gas phases along the length of the flow reactor.

Figure 9 presents a physical representation of the CSTR volume used for calculating the posterior distribution. Reactor specifications were taken from published literature and are reflective of reforming conditions associated with inline configuration with an SOFC.

The HFM for the CSTR was used to create comparison data under transient conditions. Artificial noise was added to the results of the HFM to make the data more representative of experimental testing, and to assist the calibration. The addition of this error allows the routine to aim for a small region of values at each time step as opposed to pinpointing to 


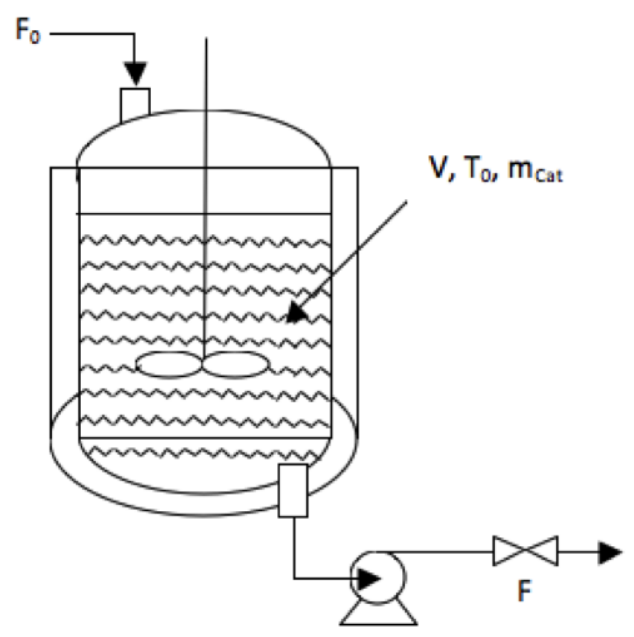

Figure 9: Simulated Reactor Environment

a single number. Using the comparison data from the HFM, the combination ROM and MCMC routine is used to solve for the posterior distribution of the discrepancy parameters. In an attempt to assist the calibration routine, initial values for the model parameters, $\theta$, were determined by conducting trials of the ROM without the addition of the discrepancy terms $(\beta=0)$. A trial and error method was employed testing sets of base model parameters until a set of concentration profiles closely resembling those of the HFM in terms of the steady state value were achieved. Starting with these values allows for the MCMC routine to operate much faster in determining the desired posterior distribution. Using the data for the HFM along with the initial parameter set from the non-discrepancy trials, the combination ROM and MCMC calibration routine is used to solve for the posterior distribution.

Success of the calibration is attained by meeting two key criteria: coverage of the HFM results by the realizations of the ROM and convergence of the posterior distribution. Coverage of the HFM data is verified by overlaying the data with realizations of the ROM using a sample of parameter sets taken from the posterior. The collective of these forms a spectrum of possible outcomes achievable from the ROM, and it is desired for the comparison data to lie within this produced spectrum. Confirming a converged solution is done by performing a Student t-test comparing key statistics of subsections of the collected posterior distribution 
to those of the whole population. Employing the central limit theorem, which states that simple means are normally distributed in the limit of infinite size, the t-test uses this theorem along with t-statistics to tell how close the distributions mean is to the actual population mean, with what confidence. This defined posterior distribution will also serve to make predictions of ROMs for plug flow reactors extrapolated from this initial transient CSTR study.

In addition to the transient response, a plug flow reactor approximated through successive CSTRs was also explored to investigate the method's ability to handle propagation in space and size. This approximation of a plug flow reactor is validated by examining the sizing equations for each reactor shown in Equations 58 and 59.

$$
\begin{gathered}
V=\frac{F_{A 0} X}{\left(-r_{A}\right)} \\
F_{A 0} \frac{d X}{d V}=-r_{A}
\end{gathered}
$$

Using these, a Levenspiel plot, like the generic example presented in Figure 10a, can be drawn comparing the ratio of entrance flow rate to reaction rate against overall conversion. In this plot configuration, the area of the chart is equivalent to reactor volume. Comparing Equations 58 and 59 in terms of the Levenspiel plot, the volume of the plug flow reactor necessary to achieve a given conversion percentage is given by the integral of the curve to that point (Figure 10b); whereas, for the CSTR, the volume necessary is equal to the product of the conversion and the ratio of flow rate to reaction rate at that point (Figure 10c). So by reducing the size and increasing the overall number of CSTRs, the volume of the plug flow reactor can be properly estimated in the same way a Riemann sum is used to approximate the value of an integral (Figure 10d).

The implementation of this approximation method required both computational models to be adjusted to reflect this change in physical geometry. Realizations for the ROM of the plug flow reactor were created using the initially calibrated posterior distribution and 


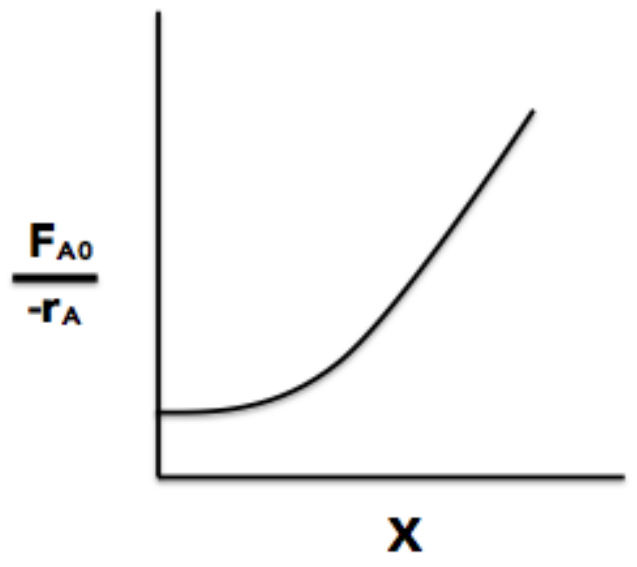

(a)

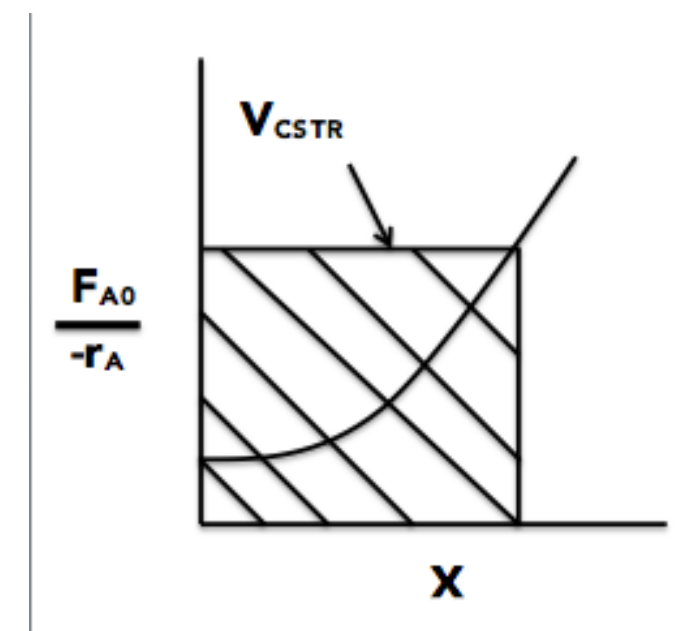

(c)

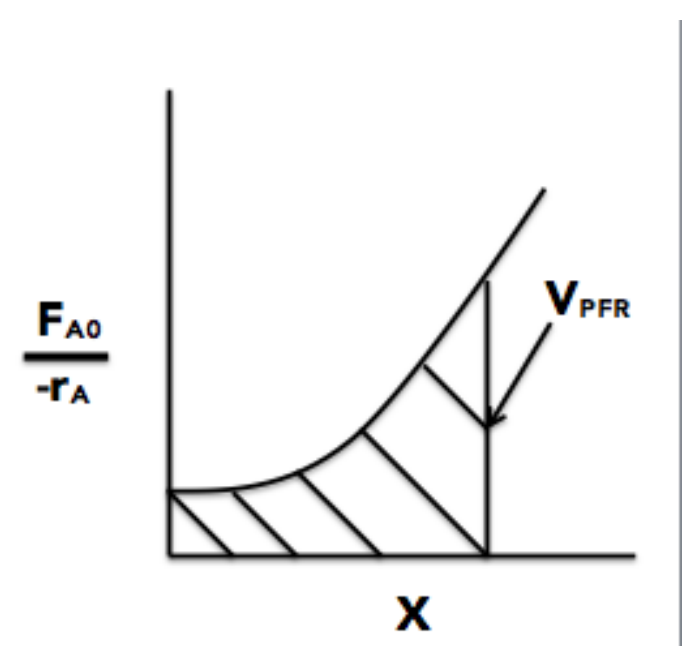

(b)

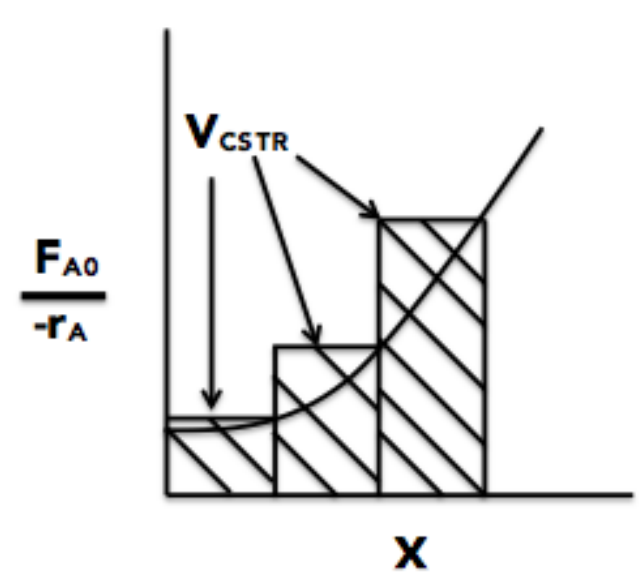

(d)

Figure 10: CSTR Approximation of PFR

compared in a similar fashion to the findings of the flow reactor HFM. For this case, only steady-state analysis were presented with key emphasis on species concentration across the length of reactor. Initially, this was demonstrated for a lab scale case in which the PFR consisted of multiple CSTRs used for demonstrating the transient response. An industrial sized SMR PFR operating at conditions far from what used from calibration was also investigated using this same posterior distribution and discretized approximation. This would ultimately test the flexibility of the calibration methodology to show the range of simulations capable of being reproduced from a single posterior distribution. 
If the realizations of the ROM at the PFR conditions are able to fully cover the HFM data along the length of the reactor bed, it will demonstrate the capability of the calibration method to extrapolate and provide solid predictions for models more detailed and complex than the conditions under which the posterior was developed, giving evidence to the claim that there exists a single set of parameters that will allow the ROM to perfectly recreate the HFM results.

\section{Results and Discussion}

\subsection{Introduction}

Following the methodology detailed in previous chapters, simulations were conducted to determine the capability of the dynamic discrepancy method for reactors on both the lab and industrial scale. Completing a successful calibration requires the meeting of two main criteria: coverage of the predictions of the HFM and convergence of the posterior distribution to a confined region in parameter space. A single posterior distribution was calibrated using the results from the lab scale CSTR as a benchmark and then applied to the ROM for both PFR simulations. Computational time was also investigated to determine if the proposed method is resourcefully more advantageous than current methods.

\subsection{Lab Scale CSTR}

Simulations for the transient CSTR response were completed following the previously described procedure. Geometry and specifications for the reactor environment are taken from previously published research by Georgis et al. given their assumptions of both a well mixed reactor and isothermal behavior[39]. This would allow for at least a qualitative comparison of the produced results.

Specifications for the initial CSTR were taken from Georgis et al.[39]. Initially the reactor is charged with an equal concentration of each gas, and the reactor is assumed to operate 
constantly at 800 degrees Kelvin. The catalyst modeled in this study is nickel mounted on magnesium, and a void fraction of 0.4 was maintained in the reactor. Steam and methane is inlet to the system at a $2: 1$ ratio $\left(\frac{S}{C}\right)$ with a variable outflow depending on the overall concentration of gas in the volume. The simulation was conducted for five seconds which was adequate time for the system to achieve a steady state solution. Table 2 summarizes the specifications and initial conditions used for the single CSTR testing.

Table 2: CSTR Specifications and Initial Conditions

\begin{tabular}{||c|c|c||}
\hline Parameter & Value & Unit \\
\hline $\mathrm{V}$ & 3.3 & $\mathrm{~L}$ \\
$\frac{S}{C}$ & 2 & - \\
$\rho_{\text {cat }}$ & 2335 & $\frac{\mathrm{kg}}{\mathrm{m}^{3}}$ \\
$\epsilon$ & 0.4 & - \\
$\dot{N}_{T o t}$ & 0.833 & $\frac{\mathrm{mol}}{\mathrm{s}}$ \\
$P_{i, 0}$ & 0.2 & $\mathrm{bar}$ \\
$\mathrm{T}$ & 800 & $\mathrm{~K}$ \\
$\mathrm{t}$ & 5 & $\mathrm{~s}$ \\
\hline
\end{tabular}

Comparison data was created using the values in Table 2 in the HFM to serve as a point of comparison for the likelihood calculation in the determining of the posterior distribution for the discrepancy parameters. Artificial noise was added to the initial results of the HFM to assist the calibration. At a signal to noise ratio (SNR) of 500, this provided a small window of values for the calibration to solve for at each time step while maintaining the appearance of a data set pulled from a realistic trial.

Sampling for the posterior was done for a high number of MCMC steps $(\sim 80,000)$ each representing a different realization of the ROM detailed in Equation 56. Coverage of the realistic data was shown by reproducing and overlaying a small set of these realizations against the findings of the HFM for each of the gas species. Shown in Figure 11, it is evident 
that the calibration routine achieved the desired result, covering the full set of realistic data at all sets. This full coverage adds validity to the idea that a ROM developed in this method is capable of making accurate predictions about the process of steam reforming. 


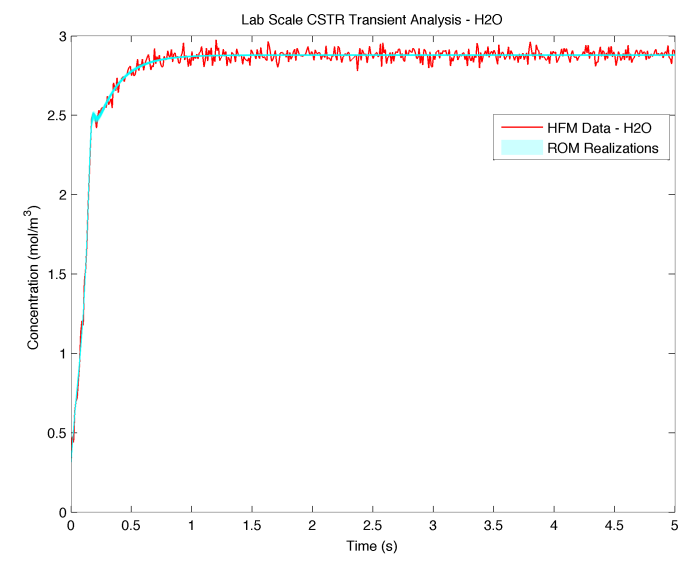

(a) $\mathrm{H}_{2} \mathrm{O}$

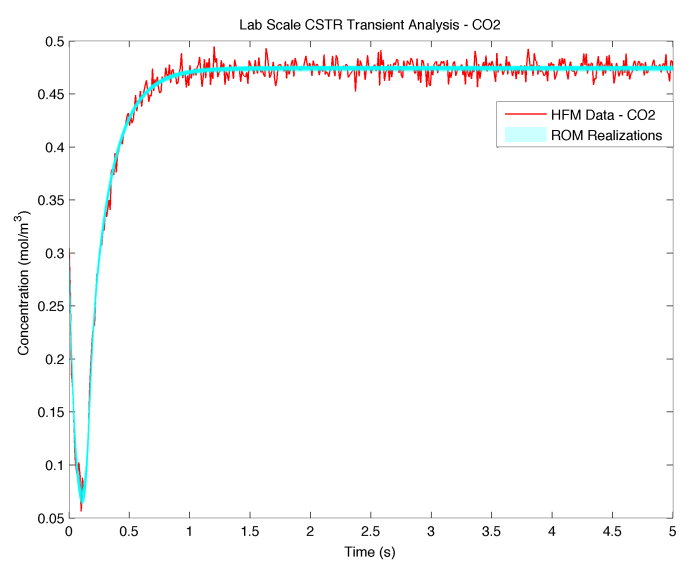

(c) $\mathrm{CO}_{2}$

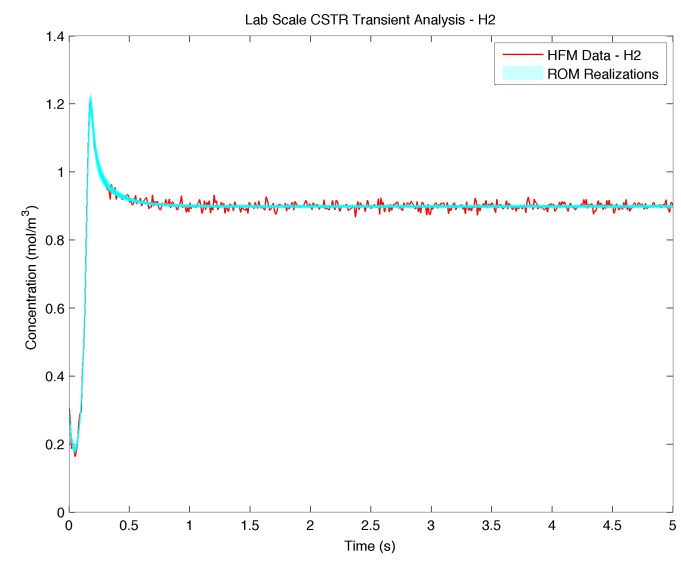

(e) $\mathrm{H}_{2}$

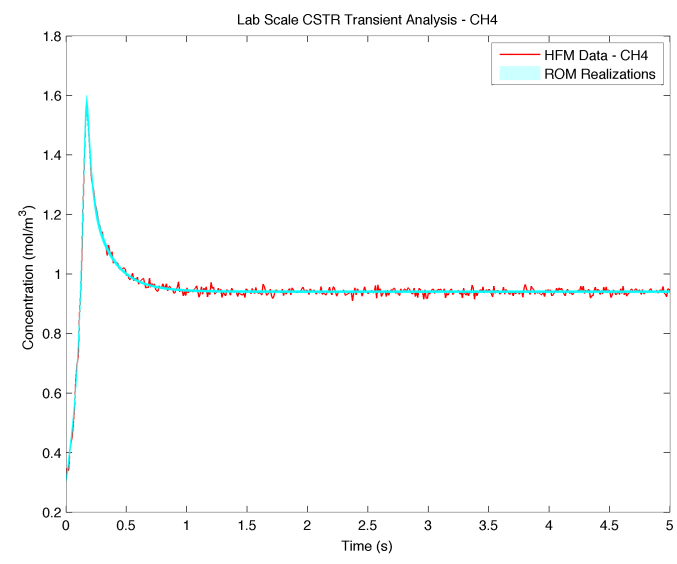

(b) $\mathrm{CH}_{4}$

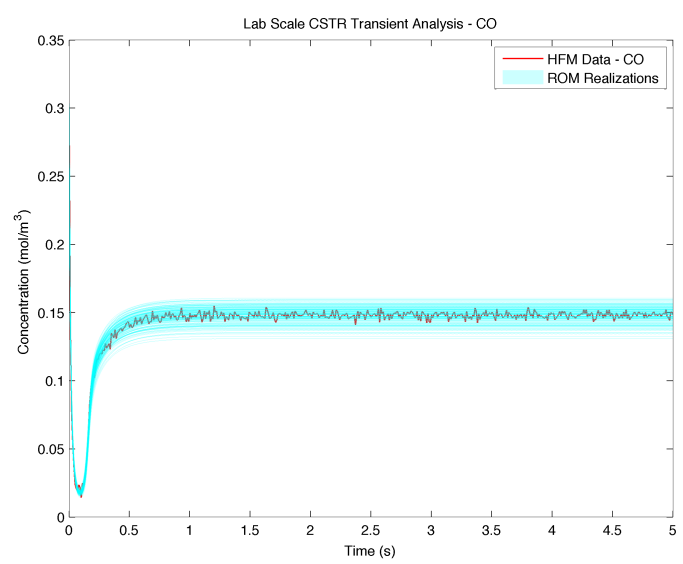

(d) $\mathrm{CO}$

Figure 11: CSTR Reality Data vs. ROM Realizations 


\subsection{Convergence Determination}

Determining a converged solution requires a more analytic approach, but there are several graphical indicators that can be used to estimate the number of MCMC steps necessary for the convergence criteria. Examining the value of the likelihood as a function of the number of MCMC step can provide a solid indication as to when the desired region in parameter space is discovered. For the calculated posterior distribution, this functional dependency is depicted in Figure 12.

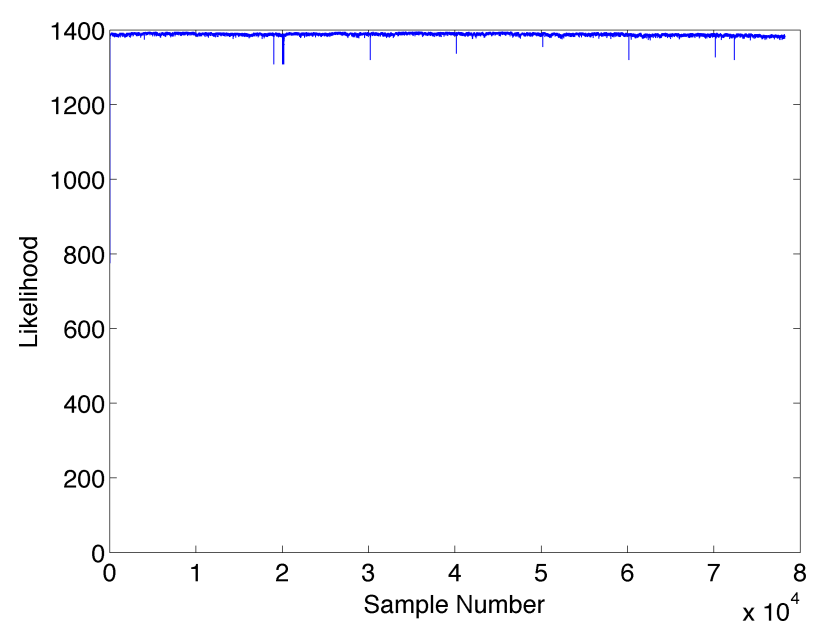

(a)

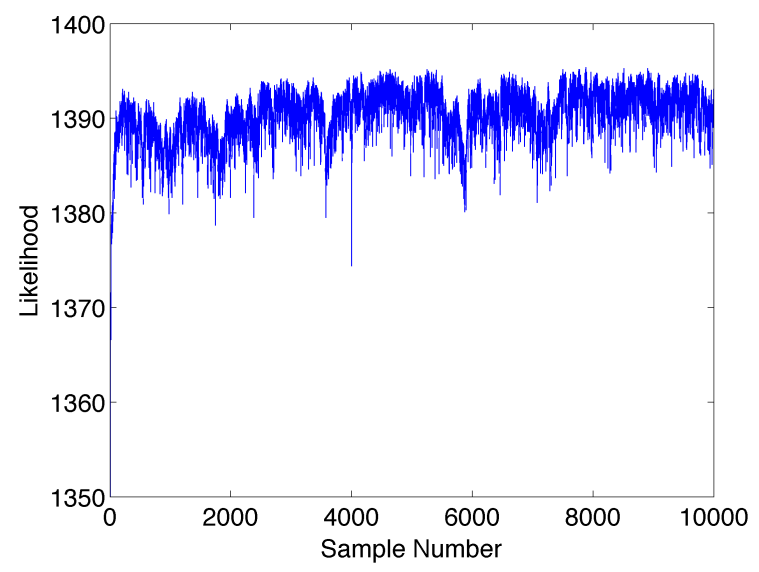

(b)

Figure 12: Likelihood Calculation for MCMC Sampling

After an expected, unsteady "burn in" period of roughly 7,000 samples (Figure 12b), the value of the likelihood settles and remains constant for a high number of MCMC steps. This shows to a certain degree that the random walk of the routine has landed and maintained in a bounded region producing similar results and likelihood values; however, this is only a possible indicator of convergence in the posterior distribution.

One way of visualizing the posterior data is through the creation of bivariate scatter plots. These give an indication of values for variables within the discrepancy function and allow for inferences to be made about the relationship between pairs of associated terms. Naturally, at large numbers of parameters this becomes an ineffective manner to examine the 
resulting distribution (as is the case with the values of the $\beta$ vector), but can be enlisted to examine the behavior of the model parameters $\theta$. Figure 13 shows bivarate scatter plots for each combination of the model parameters that constitute the $\theta$ vector. From these it is clear to see the "movement" of the MCMC routine from the initial values selected (designated by the red dots).

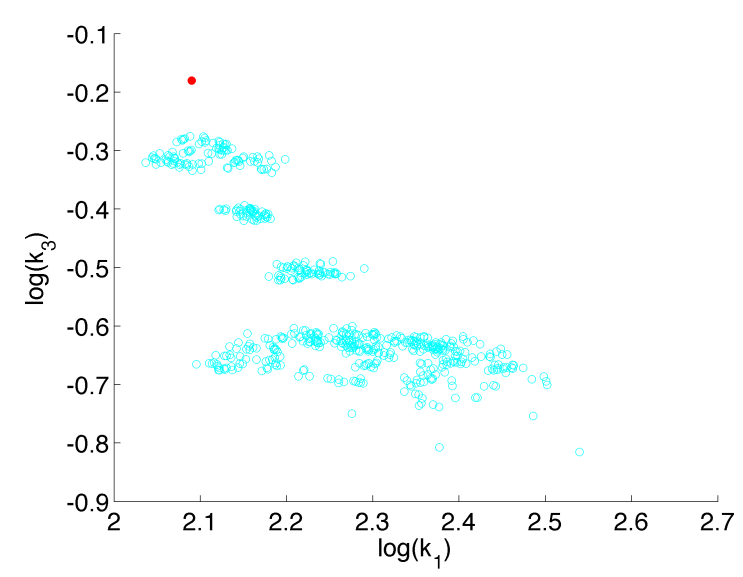

(a)

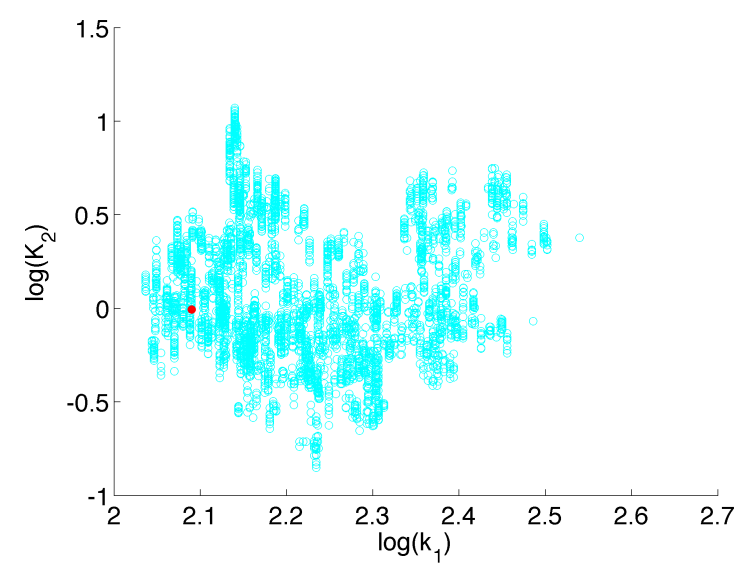

(c)

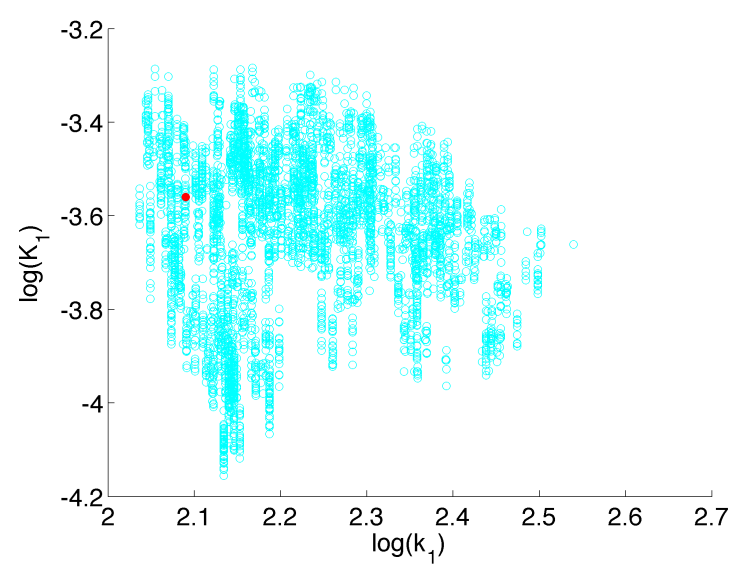

(b)

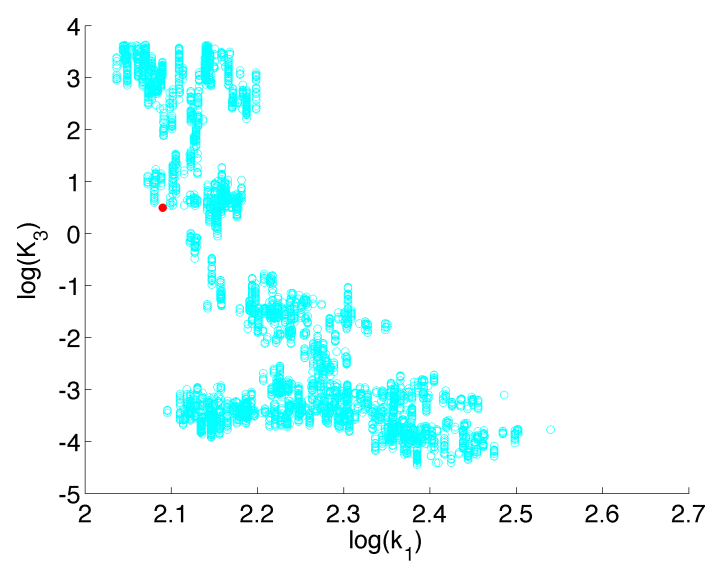

(d) 


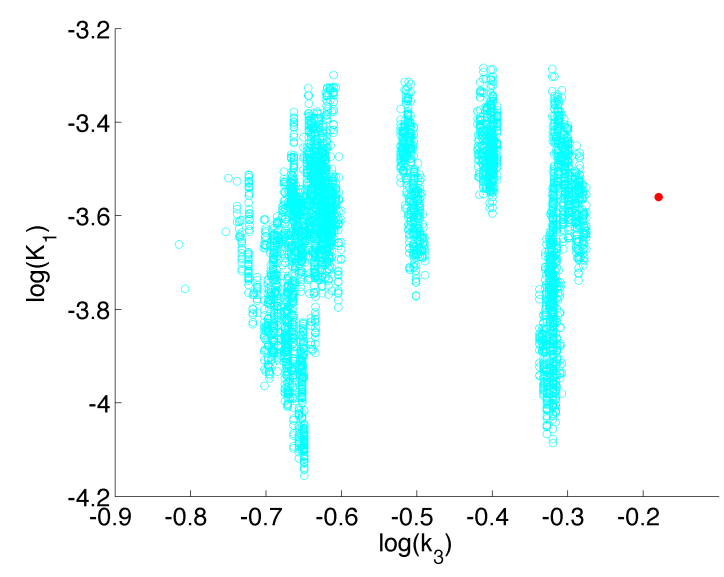

(e)

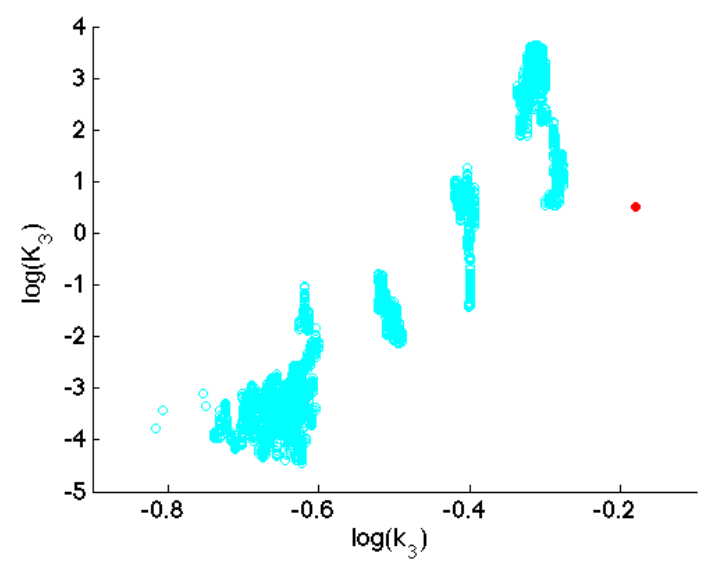

(g)

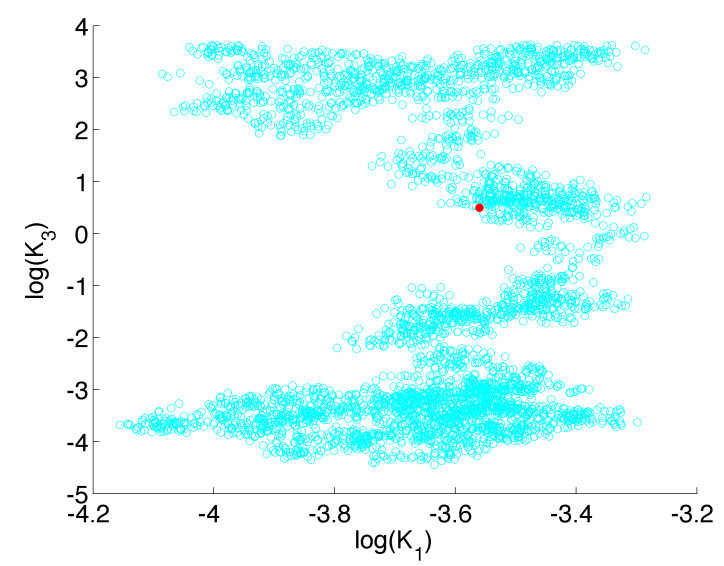

(i)

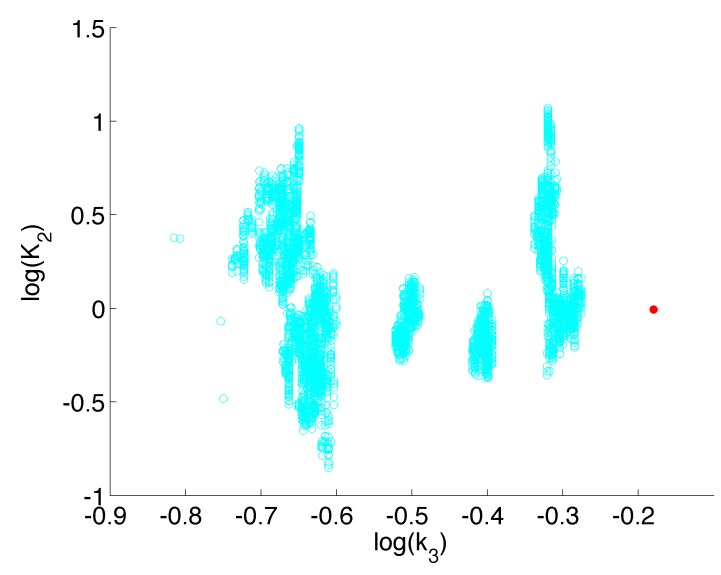

(f)

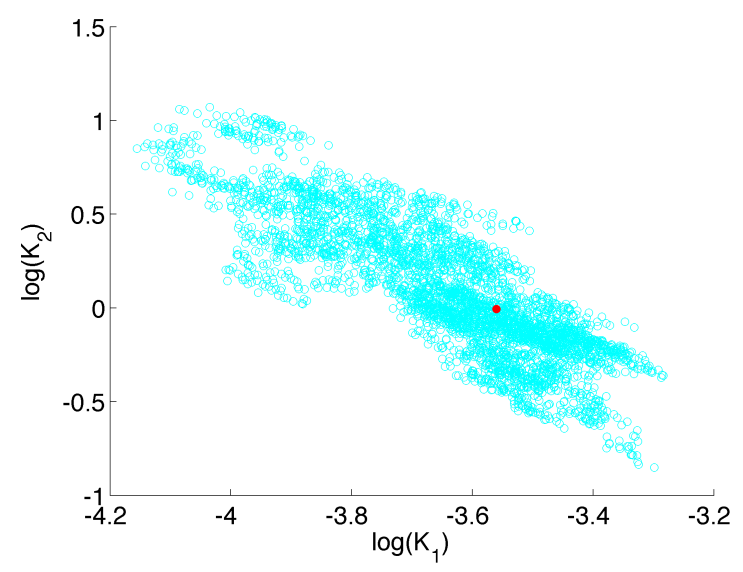

(h)

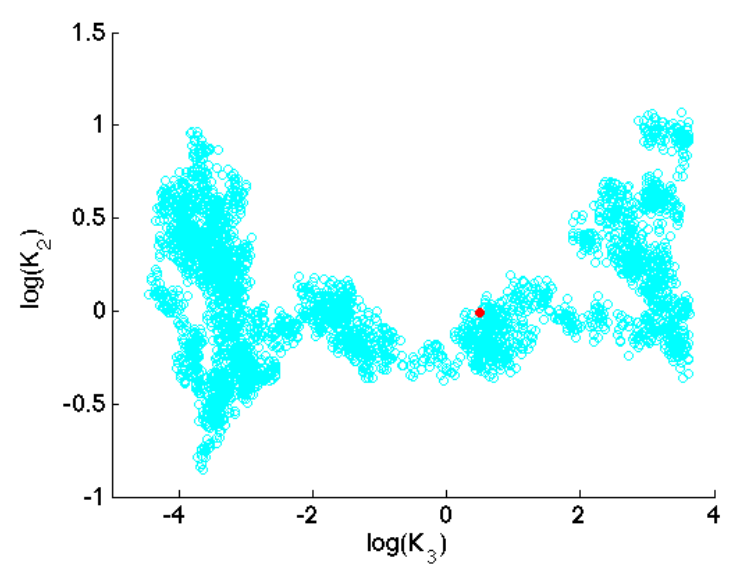

(j)

Figure 13: Bivariate Scatter for $\theta$ Posterior Distributions 
Confirmation of a converged posterior distribution was verified statistically through the use of a batch means test. Testing takes the total number of post burn in samples on the posterior distribution, $M$, and divides the data into a number of bins, $a$, each with an equal number of data points, $b$ such that:

$$
M=a b
$$

The mean for each bin was then computed and compared to the global mean for the data set to determine if there is a statistically significant difference between the two. Comparison was done on a $95 \%$ confidence interval $(\alpha=0.05)$ and a burn in of $10 \%$ of the total number of samples was used. The bins for testing were set up so that the total number was equal to the number of data points per bin $(a=b)$. Table 3 summarizes the specifications used in the convergence testing.

Table 3: Convergence Testing Conditions

\begin{tabular}{||c|c|c||}
\hline Parameter & Value & Unit \\
\hline$C I$ & $95 \%$ & - \\
$\alpha$ & 0.05 & - \\
$a$ & 265 & bins \\
$b$ & 265 & points \\
$n_{B I}$ & 8000 & samples \\
\hline
\end{tabular}

Based on this analysis, the posterior distributions for all five model parameters and the $270 \beta$ values used within the discrepancy were converged showing no statistically significant difference between the bin means and the overall population mean. Using the initial guess from the non-discrepancy trial allowed for the calibration routine to achieve a converged solution in fewer steps of the MCMC routine than what would be expected starting from an arbitrary point. Table 4 shows the statistical findings of a small sample set of results for the model and $\beta$ parameters. 
Table 4: Convergence Testing Conditions

\begin{tabular}{|c|c|c|c||}
\hline Variable & $\mu$ & $\bar{X}_{\text {Batch }}$ & $\sigma$ \\
\hline$k_{1_{0}}$ & 2.2346 & 2.2347 & 0.1001 \\
$k_{3_{0}}$ & -0.5345 & -0.5344 & 0.1330 \\
$K_{1_{0}}$ & -3.6076 & -3.6072 & 0.1641 \\
$K_{2_{0}}$ & 0.0049 & 0.0043 & 0.3551 \\
$\beta_{34}$ & 0.4915 & 0.4912 & 0.6068 \\
$\beta_{10}$ & -0.3063 & -0.3056 & 0.7224 \\
$\beta_{26}$ & -0.5878 & -0.5865 & 0.7235 \\
$\beta_{149}$ & -1.3494 & -1.3485 & 0.7758 \\
$\beta_{27}$ & 1.5055 & 1.5060 & 0.8564 \\
\hline
\end{tabular}

Because of this high rate of convergence, it can be confirmed that the MCMC routine was run sufficiently long enough to achieve a converged posterior distribution for all of the associated parameters.

\subsection{Lab Scale PFR}

Given the results of the initial trials of a single CSTR, it was desired to expand the scope of the project to include simulation within a PFR. A methodology was explored to simplify the problem by discretizing the reactor volume and allowing each of these sections to behave similar to that of the initial CSTR. This idea was examined on two different flow reactor cases: an idealized geometry comprised of multiple sections each identical to the initial CSTR, and an industrial sized reactor detailed in previous research. The posterior distribution for the CSTR trial was implemented into the ROM for each flow reactor, so only an investigation into the coverage of the reality is necessary. Steady state gas concentration along reactor length was of primary concern, ignoring the transient behavior for each individual discretization.

This idealized condition, referred to as the lab scale PFR, aims to examine the ability of 
this modeling method as the simulation moves further from the point of calibration. Each section of the reactor volume has the same initial conditions listed in Table 2 and a sufficient number of reactors were aligned until an equilibrium was achieved for the gas concentration levels in the spatial direction. Table 5 summarizes the overall specifications for the PFR used in this idealized case.

Table 5: Lab Scale PFR Specifications and Initial Conditions

\begin{tabular}{||c|c|c||}
\hline Parameter & Value & Unit \\
\hline$V_{\text {Tot }}$ & 15 & $\mathrm{~L}$ \\
$N_{r}$ & 20 & - \\
$\frac{S}{C}$ & 2 & - \\
$\rho_{\text {cat }}$ & 2335 & $\frac{k g}{m^{3}}$ \\
$\epsilon$ & 0.4 & - \\
$\dot{N}_{T o t}$ & 0.833 & $\frac{m o l}{s}$ \\
$P_{i, 0}$ & 0.2 & $\mathrm{bar}$ \\
$\mathrm{T}$ & 800 & $\mathrm{~K}$ \\
$\mathrm{t}$ & 5 & $\mathrm{~s}$ \\
\hline
\end{tabular}

Figure 14 gives the results of the implementation of the posterior distribution into the ROM for the lab scale PFR. Compared to the HFM data for the reactor, the results of the ROM provide full coverage across the full length of the reactor. As expected, the further the reactor environment gets from the calibration point, the wider the spectrum of realizations becomes, primarily for the products, but because the reality still lies within the central region of this band, it is still adequate for a first round of calibration. 


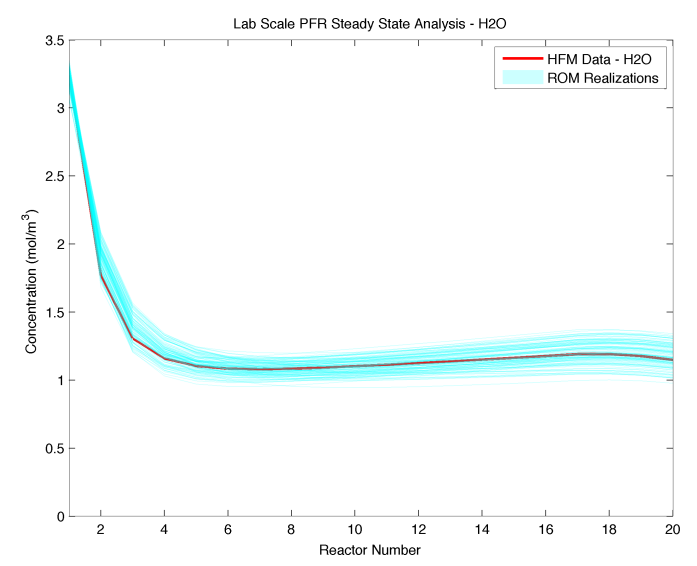

(a) $\mathrm{H}_{2} \mathrm{O}$

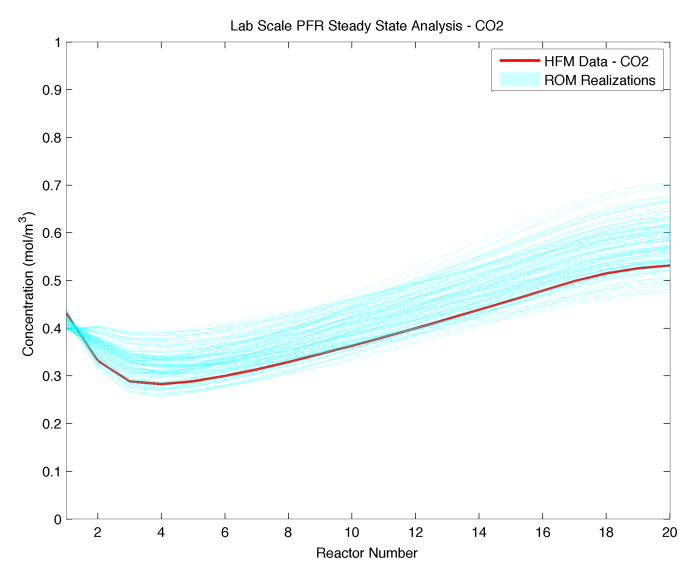

(c) $\mathrm{CO}_{2}$

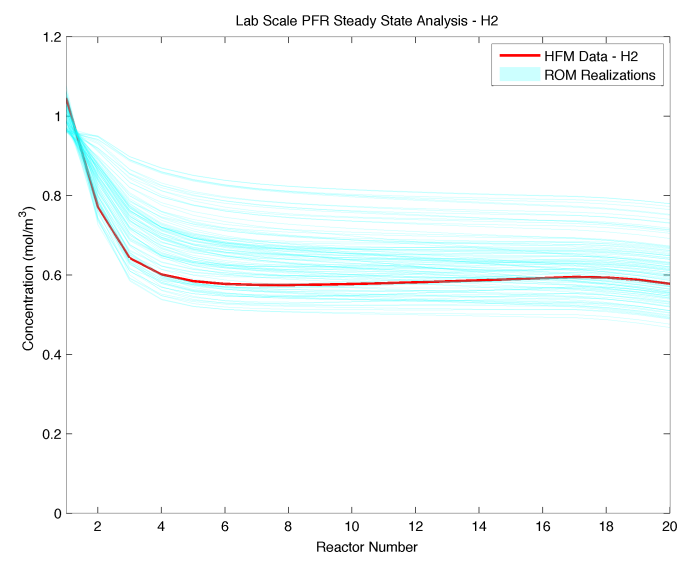

(e) $\mathrm{H}_{2}$

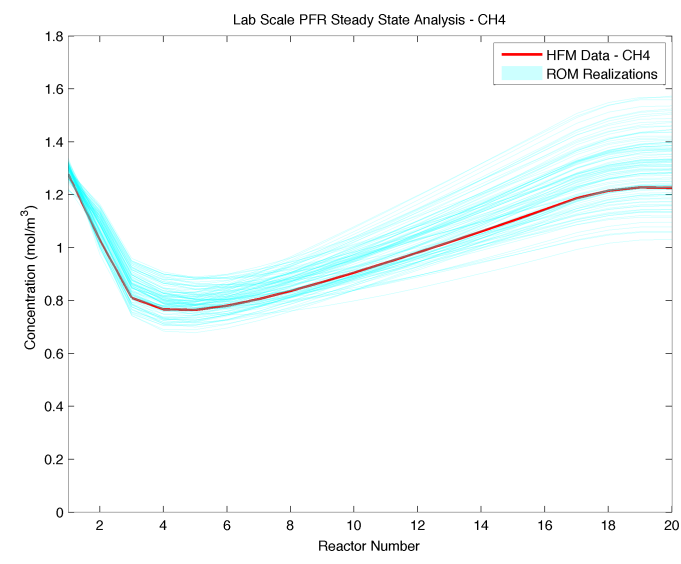

(b) $\mathrm{CH}_{4}$

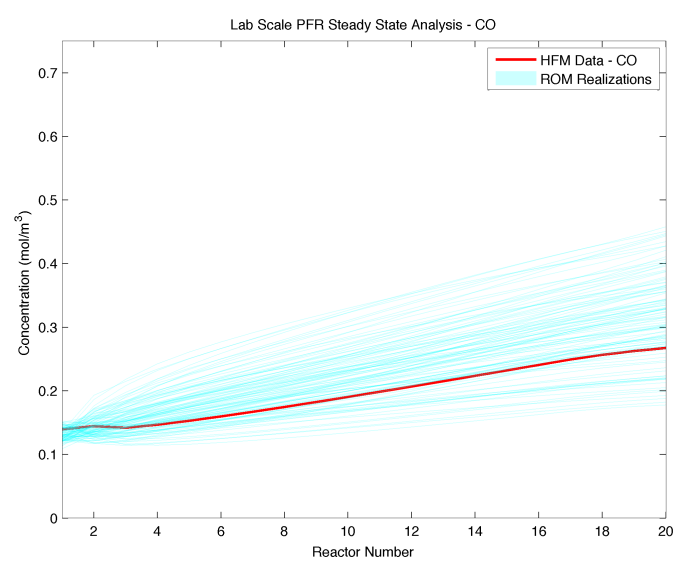

(d) $\mathrm{CO}$

Figure 14: Lab Scale PFR Reality Data vs. ROM Realizations 
This breadth of uncertainty can be reduced by restarting the calibration using the parameters associated with one of the realizations most aligned with the reality data and operating the MCMC sampling at a much small step size, but was not completed as part of this study.

\subsection{Industrial Scale PFR}

The final scenario tested with this posterior distribution looked at the operation of an industrial scale reactor used for mass production of methane reformation. The geometry of the reactor is detailed in simulation work done by Nandasana, et, al. for a multi-tube flow reactor with external heating.[40] For the purpose of this study, only a single tube was investigated and assumed to be representative of all those in the reactor system. Table 6 summarizes the specifications used in the analysis. The total volume was discretized into 24 volumes $\left(n_{r}\right)$ representing $0.5 \mathrm{~m}$ of the overall length of the reactor. Similar to the previous simulations, a well mixed and isothermal condition was imposed on the individual volumes.

Table 6: Industrial Scale PFR Specifications and Initial Conditions

\begin{tabular}{||c|c|c||}
\hline Parameter & Value & Unit \\
\hline $\mathrm{L}$ & 12 & $\mathrm{~m}$ \\
$\mathrm{~d}$ & 0.0795 & $\mathrm{~m}$ \\
$\frac{S}{C}$ & 4.8 & - \\
$\rho_{b}$ & 946.8 & $\frac{\mathrm{kg}}{\mathrm{m}^{3}}$ \\
$\epsilon$ & 0.605 & - \\
$\dot{N}_{\text {Tot }}$ & 5.434 & $\frac{\mathrm{mol}}{\mathrm{s}}$ \\
$n_{r}$ & 24 & - \\
$n_{\text {tube }}$ & 176 & - \\
$P_{i, 0}$ & 0.2 & $\mathrm{bar}$ \\
$\mathrm{T}$ & 800 & $\mathrm{~K}$ \\
\hline
\end{tabular}

Figure 15 presents the comparisons of the realizations of the ROM to the realistic data for 
each of the gas concentrations in the industrial reactor. The behavior of the concentration profiles is similar to that of the lab scale PFR showing the coverage of the reality and the increasing uncertainty in the prediction further from the origin. 


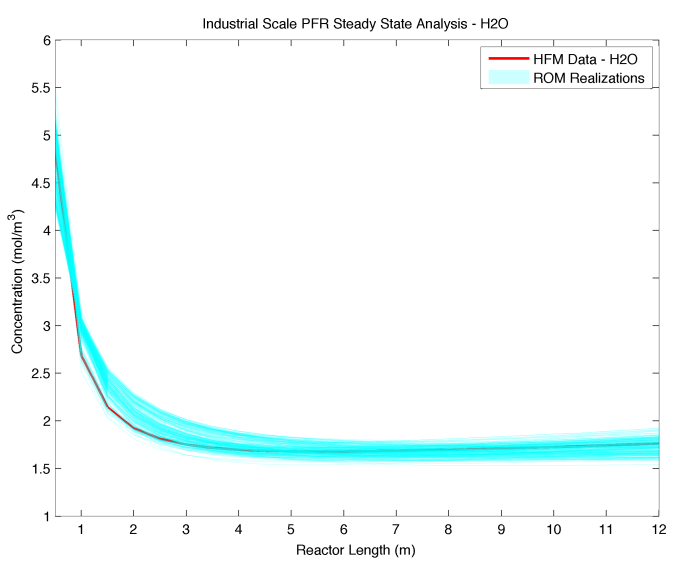

(a) $\mathrm{H}_{2} \mathrm{O}$

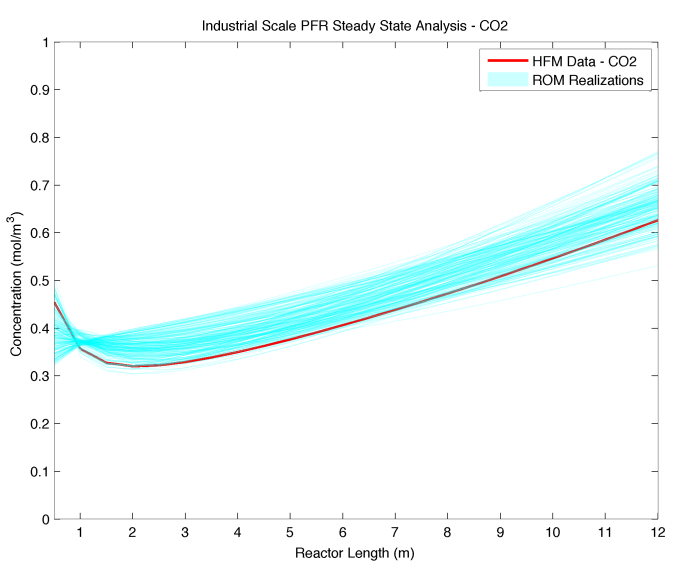

(c) $\mathrm{CO}_{2}$

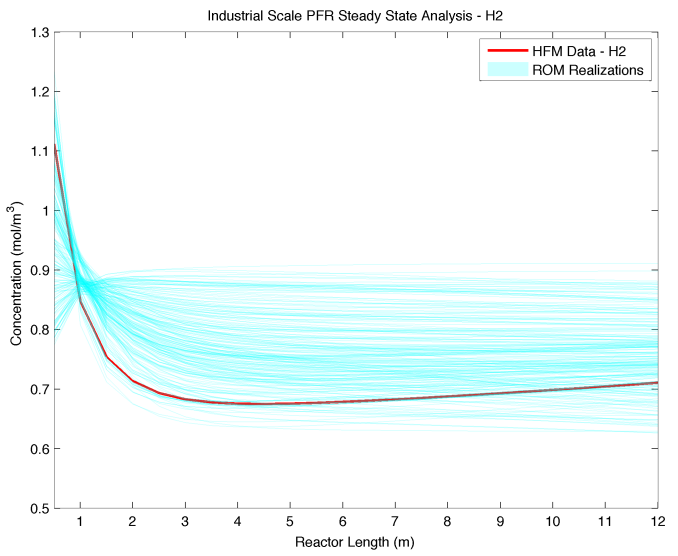

(e) $\mathrm{H}_{2}$

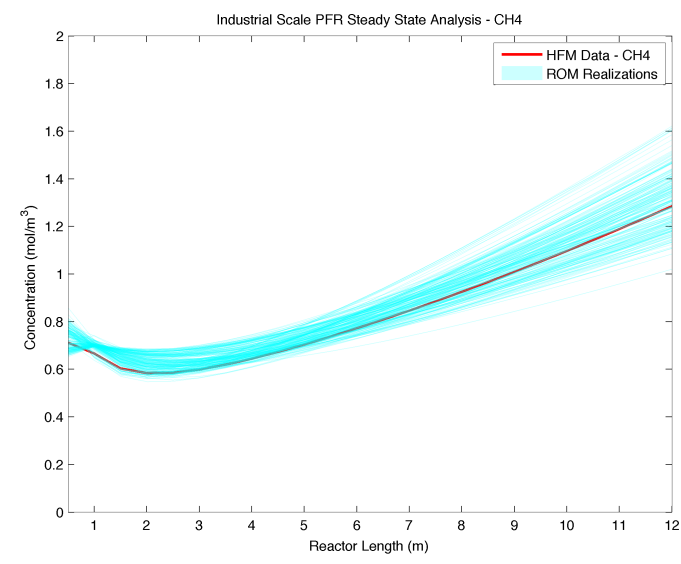

(b) $\mathrm{CH}_{4}$

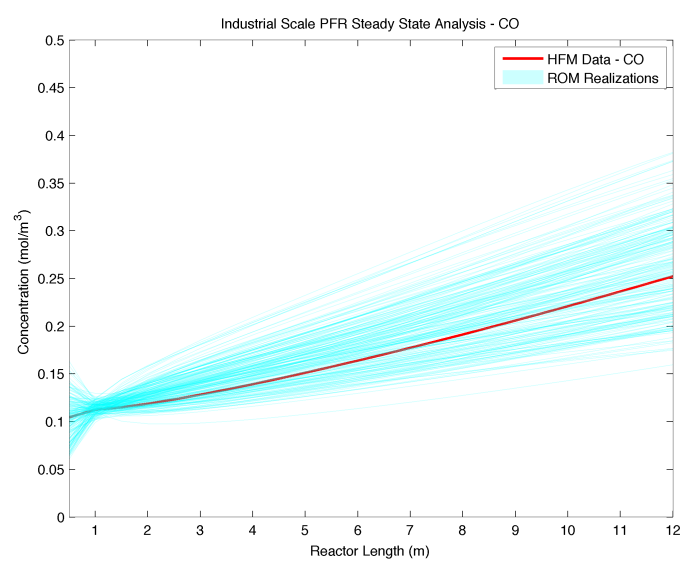

(d) $\mathrm{CO}$

Figure 15: Industrial Scale PFR Reality Data vs. ROM Realizations 
However, because the exact conditions for the calibration do not exist in this simulation there is a higher level of uncertainty even in the leading edge of the reactor system. The ability of the posterior distribution to maintain coverage of the comparison data for a reactor model much more robust adds additional validity to the idea that a suitable ROM can be developed for this system.

\subsection{Impact of Discrepancy Terms}

In order to better understand the importance that the discrepancy term has on the reduced model and calibration, simulations were conducted using only the posterior distributions of the model parameters $(\beta=\delta=0)$. Figure 16 shows the results of this non-discrepancy trial for the single CSTR system. It is clear from these realizations that the importance of the discrepancy term is primarily in allowing the ROM to adapt to a system's transient response. With the exception of the concentration of carbon monoxide, all of the spectra fall well short of covering the calibration data, and show inconsistency in providing steady state coverage. From this it can be concluded that inclusion of the discrepancy is critical for both model validation and applications involving transient response. 


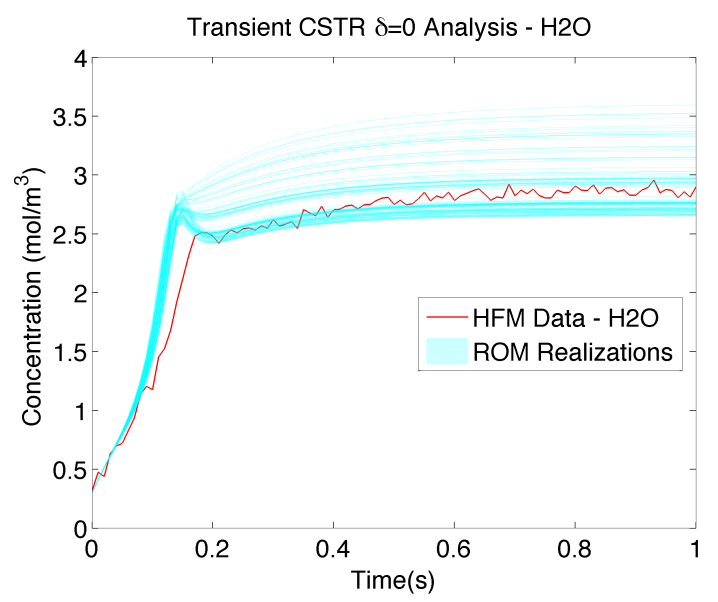

(a) $\mathrm{H}_{2} \mathrm{O}$

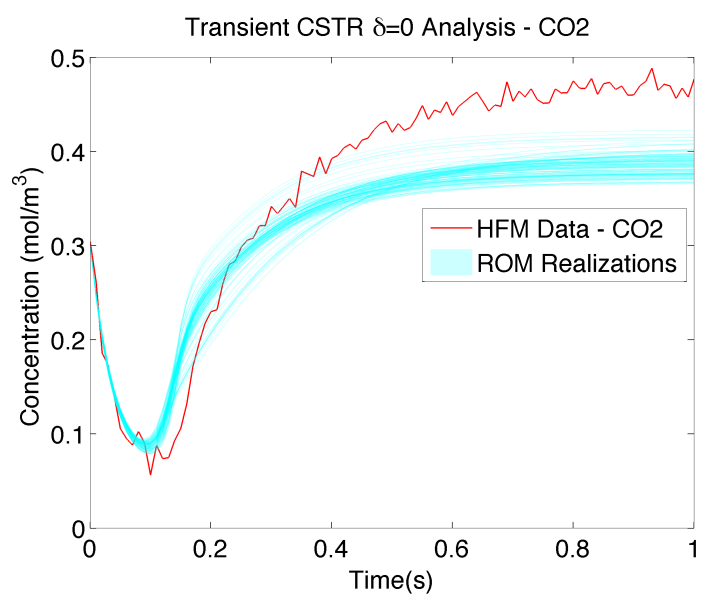

(c) $\mathrm{CO}_{2}$

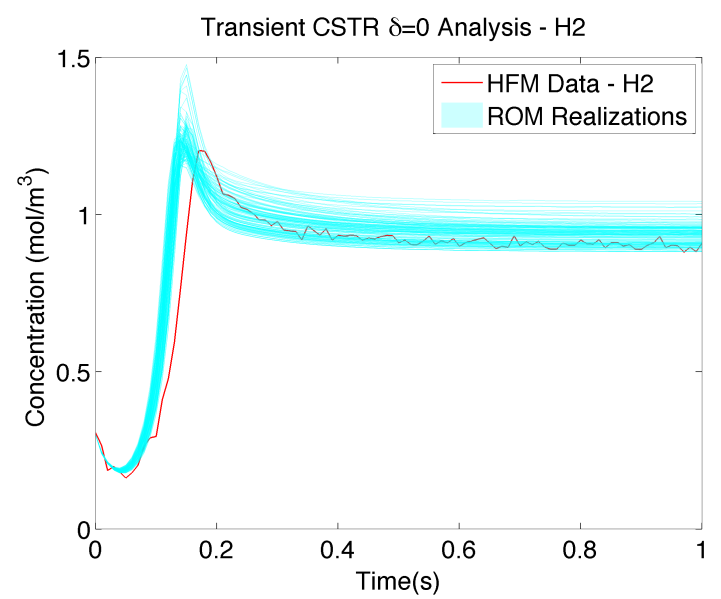

(e) $\mathrm{H}_{2}$

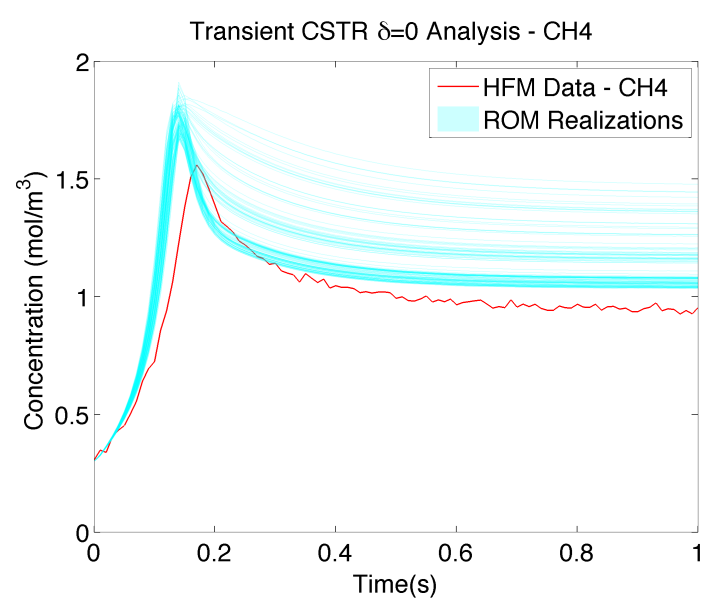

(b) $\mathrm{CH}_{4}$

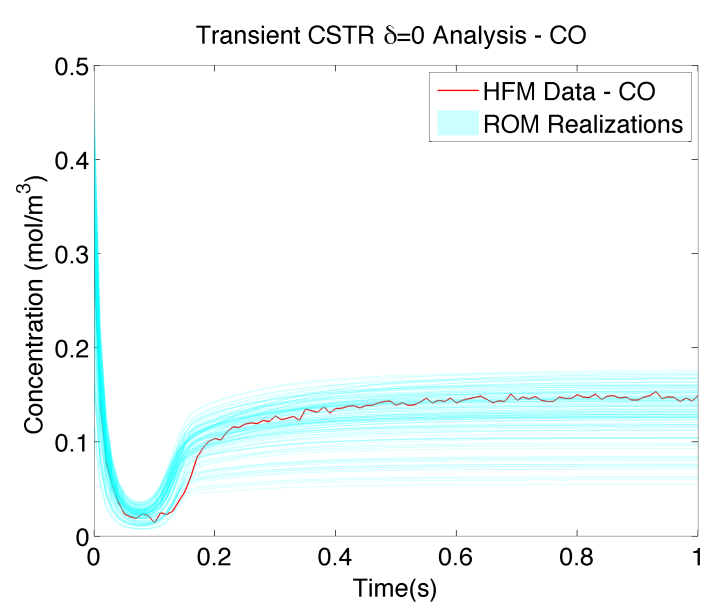

(d) $\mathrm{CO}$

Figure 16: ROM CSTR Transient Response without Discrepancy 
The non-discrepancy trials show just how important the inclusion of this term is to the reduced order modeling scheme. In addition, this demonstrates its necessity for applications requiring transient analysis (i.e. reactor control) and adds confidence to attempt of applying this technique to even more robust reaction networks or other applications that could have potentially larger system reductions, even when only a steady state analysis is required. This is the main advantage of this discrepancy function: the possibility of taking massive, complex engineering problems and reducing them to their simplest possible nature without a loss in quality of results. This would allow for the development of a ROM for process optimization and control that would be accurate, yet computationally tractable.

\subsection{Temporal Analysis}

In addition to being able to reproduce data results, it was desired for the application of the Bayesian modeling to show a distinct reduction in necessary computational time though not absolutely crucial. Because of a lack of accurate time data in previous publications, this was tested by examining the run time for producing the realistic data in the high fidelity model compared to the average time for producing a single realization of the ROM. This was achieved using MATLAB's TM built in "tic" and "toc" function in the single trials of the HFM and through the recorded computational time from the Spruce Knob Computing Cluster for the ROM. The results of the time study are summarized in Table 7.

Table 7: Simulation Times for Reactor Trials

\begin{tabular}{||c|c|c||}
\hline & Average ROM Run Time (s) & HFM Run Time (s) \\
\hline CSTR & 1.356 & 1.311 \\
\hline Lab Scale PFR & 23.508 & 4.158 \\
\hline Ind. Scale PFR & 145.488 & 29.065 \\
\hline
\end{tabular}

From the results presented in Table 7 , it is clear that as the complexity of the ROM grows the necessary computational time begins to greatly exceed that of the HFM. Two key 
issues must be taken into account when examining the run time of the ROM. Firstly, the run times presented for the ROM is determined by averaging the total reported run time by the 500 samples used to create the spectrum of results presented. This takes into account the creation of new input files based on the current set of discrepancy parameters which has no real bearing on the time needed to run the actual model. Over 500 trials, this would increase the total run time slightly, but not so much as to change the trend in the values presented. Additionally, the ROM makes use of a MATLAB ${ }^{\mathrm{TM}}$ executable files (MEX) to communicate with a $\mathrm{C}+{ }^{\mathrm{TM}}$ compiler to calculate the discrepancy at each time interval. Any hindrances in executing the MEX file due to complications in communication between to the two computing software would also not be the fault of the actual model.

However, the fact that the ROM run times are longer than its respective counterparts is not entirely discouraging. Earlier applications of this method for smaller model reductions showed a much larger margin for run time. This trend indicates that there exists a tipping point in which this reduced order modeling would be the better option. Applications to more robust computational problems will demonstrate the ability of this method to vastly improve computational times as compared to standard methods. While this simplistic problem produces run times on the same order of magnitude between the two models, increasing the overall order of the problem would favor this reduction method given that having a greater number of differential equations would be more demanding as compared to a minimum number of ODEs each embedded with a less complex algebraic problem. Additionally, finding ways to optimizing on the number of calibrated parameters in each study would drastically reduce this time, especially for the larger, more complex geometries. For online problems, some of this computation could be done offline. Then depending on the operation point, the appropriate realizations could be selected. 


\section{Conclusions}

Based on the results for the detailed reactor simulations, several key conclusions of the proposed method can be drawn. Most generically, the application of the dynamic discrepancy coupled with Bayesian calibration is capable of developing a ROM suitable for producing accurate predictions for SMR modeling on both the lab and industrial scale. The first round calibrations shown in Figures 11, 14, and 15 clearly indicate the method's ability to cover the HFM results. Coupled with the results of the convergence testing, this reaffirms the idea that there exists a single set of parameters allowing for full replication of the HFM data.

Upscaling the model in both size and complexity demonstrate the ability of this modeling method to apply to a wide range of reactor specifications using only a single calibrated set of posterior data leaving potential for the use of this for both reactor design and process optimization.

Investigations into computational time indicate that as the complexity of the reactor increases the application of the methodology in this purpose becomes slightly more taxing, but in comparison with time studies of other, smaller reductions modeled with this method, this indicates that there is a very feasible sized network reduction in which this scheme would prove more advantageous than current simulation strategies. 


\section{Acknowledgements}

I would like to personally thank the following people for the help and support throughout this endeavor:

- Dr. David Mebane for serving as my advisor providing academic insight and encouragement

- Dr. Fernando Lima for acting as a co-advisor and providing technical support on all the chemical engineering facets of this endeavour

- K. Sham Bhat for the use of the MCMC computational routine and making himself available for questioning on its use

- My parents, James and Janet Ford, for their love and support throughout this project and the entirety of my collegiate career

- My lab group for providing a supportive environment in which we all have been able to achieve success 


\section{References}

[1] Jianguo Xu and Gilbert Froment. Methane steam reforming, methanation and water-gas shift: I intrinsic kinetics. AIChE Journal, 35(1):88-96, 1989.

[2] Aylin Çiğdem Köne and Tayfun Büke. Forecasting of $\{\mathrm{CO} 2\}$ emissions from fuel combustion using trend analysis. Renewable and Sustainable Energy Reviews, 14(9):2906 2915, 2010.

[3] Esmail M. A. et. al. Mokheimer. On the Modeling of Steam Methane Reforming. Journal of Energy Resources Technology, 137:12001-1 - 12001-11, 2015.

[4] John N. Armor. The multiple roles for catalysis in the production of $\{\mathrm{H} 2\}$. Applied Catalysis A: General, 176(2):159 - 176, 1999.

[5] Pegah Ghanbari Bavarsad. Energy and exergy analysis of internal reforming solid oxide fuel cell-gas turbine hybrid system. International Journal of Hydrogen Energy, 32(17):4591 - 4599, 2007.

[6] B.C.R. Ewan and R.W.K. Allen. A figure of merit assessment of the routes to hydrogen. International Journal of Hydrogen Energy, 30(8):809 - 819, 2005.

[7] George W. Crabtree, Mildred S. Dresselhaus, and Michelle V. Buchanan. The hydrogen economy. Physics Today, 57(12):39 - 44, 2004.

[8] J. K. Rajesh, Santosh K. Gupta, G. P. Rangaiah, and Ajay K. Ray. Multiobjective optimization of steam reformer performance using genetic algorithm. Industrial $\&$ Engineering Chemistry Research, 39(3):706-717, 2000.

[9] Yuzhang Wang, Fumihiko Yoshiba, Makoto Kawase, and Takao Watanabe. Performance and effective kinetic models of methane steam reforming over ni/ysz anode of planar \{SOFC\}. International Journal of Hydrogen Energy, 34(9):3885 - 3893, 2009. 
[10] Anna Sciazko, Yosuke Komatsu, Grzegorz Brus, Shinji Kimijima, and Janusz S. Szmyd. A novel approach to the experimental study on methane/steam reforming kinetics using the orthogonal least squares method. Journal of Power Sources, 262(0):245 - 254, 2014.

[11] W. Sangtongkitcharoen, S. Assabumrungrat, V. Pavarajarn, N. Laosiripojana, and P. Praserthdam. Comparison of carbon formation boundary in different modes of solid oxide fuel cells fueled by methane. Journal of Power Sources, 142(1-2):75 - 80, 2005.

[12] S. Assabumrungrat, N. Laosiripojana, and P. Piroonlerkgul. Determination of the boundary of carbon formation for dry reforming of methane in a solid oxide fuel cell. Journal of Power Sources, 159(2):1274 - 1282, 2006.

[13] Jun Shu, Bernard P.A. Grandjean, and Serge Kaliguine. Methane steam reforming in asymmetric pd- and pd-ag/porous ss membrane reactors. Journal of Applied Catalysis A: General, 119(2):305-325, 1994.

[14] Sang Moon Lee and Sung Chang Hong. Effect of palladium addition on catalytic activity in steam methane reforming over ni-ysz porous membrane. International Journal of Hydrogen Energy, 39(36):21037 - 21043, 2014.

[15] Giuseppe Barbieri and Francesco P. Di Maio. Simulation of the methane steam reforming process in a catalytic pd-membrane reactor. Industrial $\&$ Engineering Chemistry Research, 36(6):2121-2127, 1997.

[16] S.I. Prokopiev, Yu.I. Aristov, V.N. Parmon, and N. Giordano. Intensification of hydrogen production via methane reforming and the optimization of $\mathrm{h} 2$ :co ratio in a catalytic reactor with a hydrogen-permeable membrane wall. International Journal of Hydrogen Energy, 17(4):275 - 279, 1992.

[17] A.M. Aitani. Processes to enhance refinery-hydrogen production. International Journal of Hydrogen Energy, 21(4):267 - 271, 1996. 
[18] Y Ding and E Alpay. Adsorption-enhanced steam-methane reforming. Chemical Engineering Science, 55(18):3929 - 3940, 2000.

[19] Kaihu Hou and Ronald Hughes. The kinetics of methane steam reforming over a ni/ $\alpha$ al2o catalyst. Chemical Engineering Journal, 82(1-3):311 - 328, 2001.

[20] James Van Hook. Methane-steam reforming. Catalysis Review. Science and engineering, 21(1):1 - 51, 1980.

[21] W. W. Akers and D. P. Camp. Kinetics of the methane-steam reaction. AIChE Journal, 1(4):471-475, 1955.

[22] J. R. H. Ross and M. C. F. Steel. Mechanism of the steam reforming of methane over a coprecipitated nickel-alumina catalyst. Chemical Society Faraday Transacitons I, 69:11 $-22,1973$.

[23] P. Münster and H.J. Grabke. Kinetics of the steam reforming of methane with iron, nickel, and iron-nickel alloys as catalysts. Journal of Catalysis, 72(2):279 - 287, 1981.

[24] Jianguo Xu and Gilbert Froment. Methane steam reforming: Ii. diffusional limitations and reactor simulation. AIChE Journal, 35(1):97 - 103, 1989.

[25] M. A. Soliman, A. M. Adris, A. S. Al-Ubaid, and S. S. E. H. El-Nashaie. Intrinsic kinetics of nickle/calcium aluminate catalyst for methane steam reforming. Journal of Chemical Technology and Biotechnology, 55(2):131-138, 1992.

[26] J. C. De Deken, E. F. Devos, and F. Froment. Steam reforming of natural gas - intrinsic kinetics, diffusional influences, and reactor design. ACS Sympoesium Series, 196:181197, 1982.

[27] D. Mogensen, J.-D. Grunwaldt, P.V. Hendriksen, K. Dam-Johansen, and J.U. Nielsen. Internal steam reforming in solid oxide fuel cells: Status and opportunities of kinetic studies and their impact on modelling. Journal of Power Sources, 196(1):25 - 38, 2011. 
[28] H. Timmermann, D. Fouquet, A. Weber, E. Ivers-Tiffée, U. Hennings, and R. Reimert. Internal reforming of methane at ni/ysz and ni/cgo sofc cermet anodes. Fuel Cells, 6(3-4):307-313, 2006.

[29] Anthony L. Lee, R. F. Zabransky, and W. J. Huber. Internal reforming development for solid oxide fuel cells. Industrial \&f Engineering Chemistry Research, 29(5):766-773, 1990.

[30] E.H. Edwin, H. Karoliussen, and R. Odegard. H2S in natural gas fuel reduces mechanical stress in solid oxide fuel cells. Electrochemical Society, 1997.

[31] H. Yakabe, T. Ogiwara, M. Hishinuma, and I. Yasuda. 3-d model calculation for planar \{SOFC\}. Journal of Power Sources, 102(1-2):144 - 154, 2001.

[32] James A. Liu. Kinetics, catalysis and mechanism of methane steam reforming. Master's thesis, Worcester Polytechnic Institute, 2006.

[33] Anna Sciazko, Yosuke Komatsu, Grzegorz Brus, Shinji Kimijima, and Janusz S. Szmyd. A novel approach to improve the mathematical modelling of the internal reforming process for solid oxide fuel cells using the orthogonal least squares method. International Journal of Hydrogen Energy, 39(29):16372 - 16389, 2014.

[34] Brian J. Reich, Curtis B. Storlie, and Howard D. Bondell. Variable selection in bayesian smoothing spline anova models: Application to deterministic computer codes. Technometrics, 51(2):pp. 110-120, 2009.

[35] Curtis B. Storlie, William A. Lane, Emily M. Ryan, James R. Gattiker, and David M. Higdon. Calibration of computational models with categorical parameters and correlated outputs via bayesian smoothing spline anova. Journal of the American Statistical Association, 110(509):68-82, 2015. 
[36] David S. Mebane, K. Sham Bhat, Joel D. Kress, Daniel J. Fauth, McMahan L. Gray, Andrew Lee, and David C. Miller. Bayesian calibration of thermodynamic models for the uptake of co2 in supported amine sorbents using ab initio priors. Phys. Chem. Chem. Phys., 15:4355-4366, 2013.

[37] Caitlin A. CAllaghan. Kinetics and Catalysis of the Water-Gas-Shift Reaction: A Microkinetic and Graph Theoretical Approach. PhD thesis, Worcester Polytechnic Institute, 2006.

[38] Ethan R. Deyle and George Sugihara. Generalized theorems for nonlinear state space reconstruction. PLoS ONE, 6(3):e18295, 032011.

[39] Dimitrios Georgis, Sujit S. Jogwar, Ali S. Almansoori, and Prodromos Daoutidis. Design and control of energy integrated $\{\mathrm{SOFC}\}$ systems for in situ hydrogen production and power generation. Computers $\&$ Chemical Engineering, 35(9):1691 - 1704, 2011. Energy Systems Engineering.

[40] Anjana D. Nandasana, Ajay K. Ray, and Santosh K. Gupta. Dynamic model of an industrial steam reformer and its use for multiobjective optimization. Industrial \& Engineering Chemistry Research, 42(17):4028-4042, 2003. 